\title{
SOBOLEV ALGEBRAS ON NONUNIMODULAR LIE GROUPS
}

\author{
MARCO M. PELOSO AND MARIA VALLARINO
}

\begin{abstract}
Let $G$ be a noncompact connected Lie group and $\rho$ be the right Haar measure of $G$. Let $\mathbf{X}=\left\{X_{1}, \ldots, X_{q}\right\}$ be a family of left invariant vector fields which satisfy Hörmander's condition, and let $\Delta=-\sum_{i=1}^{q} X_{i}^{2}$ be the corresponding subLaplacian. For $1 \leq p<\infty$ and $\alpha \geq 0$ we define the Sobolev space
\end{abstract}

$$
L_{\alpha}^{p}(G)=\left\{f \in L^{p}(\rho): \Delta^{\alpha / 2} f \in L^{p}(\rho)\right\},
$$

endowed with the norm

$$
\|f\|_{\alpha, p}=\|f\|_{p}+\left\|\Delta^{\alpha / 2} f\right\|_{p},
$$

where we denote by $\|f\|_{p}$ the norm of $f$ in $L^{p}(\rho)$.

In this paper we show that for all $\alpha \geq 0$ and $p \in(1, \infty)$, the space $L^{\infty} \cap L_{\alpha}^{p}(G)$ is an algebra under pointwise product, that is, there exists a positive constant $C_{\alpha, p}$ such that for all $f, g \in L^{\infty} \cap L_{\alpha}^{p}(G), f g \in L^{\infty} \cap L_{\alpha}^{p}(G)$ and

$$
\|f g\|_{\alpha, p} \leq C_{\alpha, p}\left(\|f\|_{\alpha, p}\|g\|_{\infty}+\|f\|_{\infty}\|g\|_{\alpha, p}\right) .
$$

Such estimates were proved by T. Coulhon, E. Russ and V. Tardivel-Nachef in the case when $G$ is unimodular. We shall prove it on Lie groups, thus extending their result to the nonunimodular case.

In order to prove our main result, we need to study the boundedness of local Riesz transforms $R_{J}^{c}=X_{J}(c I+\Delta)^{-m / 2}$, where $c>0, X_{J}=X_{j_{1}} \ldots X_{j_{m}}$ and $j_{\ell} \in\{1, \ldots, q\}$ for $\ell=1, \ldots, m$. We show that if $c$ is sufficiently large, the Riesz transform $R_{J}^{c}$ is bounded on $L^{p}(\rho)$ for every $p \in(1, \infty)$, and prove also appropriate endpoint results involving Hardy and BMO spaces.

\section{Introduction AND STATEMENT OF the MAin RESUlts}

Let $G$ be a noncompact connected Lie group. We shall denote by $\lambda$ and $\rho$ the left and right Haar measures of $G$, respectively, and by $\delta$ the modular function, i.e. $\delta=\frac{\mathrm{d} \lambda}{\mathrm{d} \rho}$. For every $p \in[1, \infty]$ and $f \in L^{p}(\rho)$ we shall denote by $\|f\|_{p}$ the norm of $f$ in $L^{p}(\rho)$.

Let $\mathbf{X}=\left\{X_{1}, \ldots, X_{q}\right\}$ be a family of left invariant vector fields which satisfy Hörmander's condition and consider the subLaplacian $\Delta=-\sum_{i=1}^{q} X_{i}^{2}$. For every $p \in(1, \infty)$, let $\Delta_{p}$ be the smallest closed extension of $\left.\Delta\right|_{C_{c}^{\infty}(G)}$ to $L^{p}(\rho)$. For every $\alpha>0$ one may define the operator $\Delta_{p}^{\alpha}$ on $L^{p}(\rho)$ which we shall always denote by $\Delta^{\alpha}$, see e.g. [28]. For every $p \in(1, \infty)$ and $\alpha \geq 0$ we define the Sobolev space

$$
L_{\alpha}^{p}(G)=\left\{f \in L^{p}(\rho): \Delta^{\alpha / 2} f \in L^{p}(\rho)\right\},
$$

2010 Mathematics Subject Classification. 46E35, 22E30, 43A15.

Key words and phrases. Sobolev spaces, Lie groups, Riesz transforms.

Both authors are partially supported by the grants PRIN 2010-11 and 2015 Real and Complex Manifolds: Geometry, Topology and Harmonic Analysis. Both authors are members of the Gruppo Nazionale per l'Analisi Matematica, la Probabilità e le loro Applicazioni (GNAMPA) of the Istituto Nazionale di Alta Matematica (INdAM). 
endowed with the norm

$$
\|f\|_{\alpha, p}=\|f\|_{p}+\left\|\Delta^{\alpha / 2} f\right\|_{p} .
$$

Throughout the paper we will often denote by $L^{p}$ the space $L^{p}(\rho)$, and when we refer to $L^{p}$-integrability, we will always mean integrability with respect to the right Haar measure $\mathrm{d} \rho$. Moreover, if the underlying group $G$ is understood from the context, we will often write $L_{\alpha}^{p}$ in place of $L_{\alpha}^{p}(G)$.

Our aim is to prove the following result.

Theorem 1.1. Let $G$ be a noncompact connected Lie group. For all $\alpha \geq 0$ and $p \in(1, \infty)$ the space $L_{\alpha}^{p} \cap L^{\infty}$ is an algebra under pointwise product. More precisely, there exists a positive constant $C_{\alpha, p}$ such that for all $f, g \in L_{\alpha}^{p} \cap L^{\infty}$, we have $f g \in L_{\alpha}^{p} \cap L^{\infty}$ and

$$
\|f g\|_{\alpha, p} \leq C_{\alpha, p}\left(\|f\|_{\alpha, p}\|g\|_{\infty}+\|f\|_{\infty}\|g\|_{\alpha, p}\right) .
$$

Theorem 1.1 will be obtained as a particular case of the following more general theorem.

Theorem 1.2. Let $G$ be a noncompact connected Lie group. Let $\alpha \geq 0, p_{1}, q_{2} \in(1, \infty]$ and $r, p_{2}, q_{1} \in(1, \infty)$ such that $\frac{1}{r}=\frac{1}{p_{i}}+\frac{1}{q_{i}}, i=1,2$. There exists a positive constant $C$ such that for all $f \in L^{p_{1}}(\rho) \cap L_{\alpha}^{p_{2}}$ and $g \in L^{q_{2}}(\rho) \cap L_{\alpha}^{q_{1}}$, we have $f g \in L_{\alpha}^{r}$ and

$$
\|f g\|_{\alpha, r} \leq C\left(\|f\|_{p_{1}}\|g\|_{\alpha, q_{1}}+\|f\|_{\alpha, p_{2}}\|g\|_{q_{2}}\right) .
$$

Given a Laplacian or a subLaplacian on a Lie group, the question of finding under which conditions the corresponding Sobolev spaces form an algebra, has a long history. It was first proved by R. Strichartz [44] in the case of the Laplacian in $\mathbb{R}^{n}$ that the Sobolev spaces $L_{\alpha}^{p}\left(\mathbb{R}^{n}\right)$ form an algebra when $\alpha p>n$. Such result was later extended by G. Bohnke in the case of a nilpotent Lie group $G$ [10], under the condition $\alpha p>Q$, where $Q$ denotes the homogeneous dimension of $G$. This result was also proved by T. Coulhon, E. Russ and V. Tardivel-Nachev [15] on any unimodular Lie group $G$ when $\alpha p>d$, where $d$ denotes the local dimension of $G$; see (2.3).

Later, T. Kato and G. Ponce [27] proved Theorem 1.1 in the case of the Laplacian in $\mathbb{R}^{n}$, which is more general than Strichartz's result since it does not rely on the Sobolev embedding. Incidentally, the same authors showed that the algebra property of the Sobolev spaces is fundamental in the theory of well-posedness of Cauchy problems for certain nonlinear differential equations.

More recently, the Sobolev algebra problem was studied for Laplacians, subLaplacians and even more general differential operators satisfying suitable assumptions on various Lie groups and Riemannian manifolds [6, 9, 15, 20]. In particular, Theorems 1.1 and 1.2 were proved in [15] in the case when $G$ is unimodular.

As already mentioned, in this paper we prove Theorems 1.1 and 1.2 in the case of a subLaplacian on any nonunimodular Lie group. The situation on a nonunimodular Lie group is considerably more complicated than in the unimodular case. Indeed we prove that in general when $G$ is nonunimodular, $L_{\alpha}^{p}(G)$ is not an algebra, even when $\alpha p>d$, see Theorem 3.3. Incidentally, the same counterexample shows that the space $L_{1}^{p}(G)$ does not embed in $L^{\infty}$ when $p>d$; see also Remark 3.4. Furthermore, since $\delta$ is not trivial, we have to deal with some technical difficulties: $\delta$ obviously appears when we make some change of variables in the integrals, and the factor $\delta^{1 / 2}$ naturally arises in the estimates of the heat 
kernel associated with $\Delta$ and its derivatives (see Subsection 2.1 below). Very often we shall work on balls of small radius where the modular function is comparable with its value at the center of the ball; but sometimes we also have to deal with the behavior of the modular function on balls of arbitrary radius. Let us mention that property (2.5) below, which gives a control of the integral of $\delta^{1 / 2}$ on balls of any radius, is crucial in the proof of our results.

The main ingredient in the proof of Theorem 1.1, is a characterization of the Sobolev norm (1.1) in terms of the $p$-integrability property of averages of differences of a function on small balls. We consider the local versions of functionals introduced by Strichartz [44, and E. M. Stein [42], respectively; see also [15] for these local versions in the unimodular case. To be more precise, for a locally integrable function $f$ and every $\alpha \in(0,1)$ we set

$$
S_{\alpha}^{\text {loc }} f(x)=\left(\int_{0}^{1}\left[\frac{1}{u^{\alpha} V(u)} \int_{|y|<u}\left|f\left(x y^{-1}\right)-f(x)\right| \mathrm{d} \rho(y)\right]^{2} \frac{\mathrm{d} u}{u}\right)^{1 / 2},
$$

and

$$
D_{\alpha}^{\mathrm{loc}} f(x)=\left(\int_{|y|<1} \frac{\left|f\left(x y^{-1}\right)-f(x)\right|^{2}}{|y|^{2 \alpha} V(|y|)} \mathrm{d} \rho(y)\right)^{1 / 2} .
$$

For $r>0$, we denote by $V(r)$ the volume of the ball centered at the origin $e$ of $G$, with respect to the right Haar measure $\rho$; see (2.1). Then, we prove the following result.

Theorem 1.3. Let $G$ be a noncompact connected Lie group and let $\alpha \in(0,1)$. Then the following properties hold:

(i) for any $p \in(1, \infty)$ there exists a positive constant $C$ such that

$$
C^{-1}\|f\|_{\alpha, p} \leq\left\|S_{\alpha}^{\text {loc }} f\right\|_{p}+\|f\|_{p} \leq C\|f\|_{\alpha, p} ;
$$

(ii) for any $p>2 d /(d+2 \alpha)$ there exists a positive constant $C$ such that

$$
C^{-1}\|f\|_{\alpha, p} \leq\left\|D_{\alpha}^{\text {loc }} f\right\|_{p}+\|f\|_{p} \leq C\|f\|_{\alpha, p} .
$$

We point out that the norm equivalence (i) of Theorem 1.3 is the main tool that we use to prove Theorem 1.2 in the case when $\alpha \in(0,1)$, while the norm equivalence (ii) provides a further characterization of the Sobolev norm for certain values of $p$ and $\alpha$. In order to prove Theorem 1.2 in the case $\alpha \in[1, \infty)$, we need to prove the $L^{p}$-boundedness of the local Riesz transforms. We consider the collection of multiindices

$$
\{1, \ldots, q\}^{m}=\left\{J=\left(j_{1}, \ldots, j_{m}\right): j_{\ell} \in\{1, \ldots, q\}, \text { for } \ell=1, \ldots, m\right\} .
$$

For every $c>0$ and $J \in\{1, \ldots, q\}^{m}$, we shall denote by $R_{J}^{c}$ the local Riesz transform of order $m$

$$
R_{J}^{c}=X_{J}(c I+\Delta)^{-m / 2}
$$

where, $X_{J}=X_{j_{1}} \ldots X_{j_{m}}$.

Then, we prove the following boundedness result for $R_{J}^{c}$, whose statement involves a Hardy type space $\mathfrak{h}^{1}(\rho)$ and a space $\mathfrak{b} \mathfrak{m} \mathfrak{o}(\rho)$, whose precise definition is given in Subsection 2.3 .

Theorem 1.4. Let $G$ be a noncompact connected Lie group. There exists $c>0$ sufficiently large such that for every $J \in\{1, \ldots, q\}^{m}$ and $m \in \mathbb{N}$, the local Riesz transform $R_{J}^{c}$ is 
bounded from the Hardy space $\mathfrak{h}^{1}(\rho)$ to $L^{1}(\rho)$, from $L^{\infty}$ to $\mathfrak{b m o}(\rho)$ and on $L^{p}(\rho)$ for every $p \in(1, \infty)$.

Given a (sub)Laplacian $\Delta$ on a Lie group the question of the $L^{p}$-boundedness of the Riesz transforms $\mathcal{R}_{j}=X_{j} \Delta^{-1 / 2}$, and of their higher order analogue $\mathcal{R}_{J}=X_{J} \Delta^{-m / 2}$, where $J \in\{1, \ldots, q\}^{m}$, has also a long and rich hystory. It is well known that the $L^{p}$-boundedness of the Riesz transforms $\mathcal{R}_{j}, j=1, \ldots, q$, is tighly connected to the equivalence of two natural definitions of homogeneous first order $L^{p}$ Sobolev spaces. The Riesz transforms $\mathcal{R}_{j}$ are known to be bounded on $L^{p}$ when the underlying group is stratified [19], nilpotent 32, of polynomial growth 2] and on certain classes of Lie groups of exponential growth [26, 31, 39, 40]. On nilpotent Lie groups the Riesz transforms of higher order $\mathcal{R}_{J}$ are also bounded on $L^{p}$ [18, but it is known that this is not always the case (see [21] for an example of nonunimodular Lie group of exponential growth where the Riesz transforms $\mathcal{R}_{J}$ of order 2 are unbounded on $L^{p}$ for every $p \in[1, \infty)$ ).

In this paper we deal only with the local Riesz transforms. The $L^{p}$ boundedness of the local Riesz transforms Theorem 1.4 is known to hold on nonamenable Lie groups [30] and on every Lie group when $\Delta$ is a complete Laplacian [36]. Thus, it is certainly an expected result, and maybe considered "folklore" by many. However, to the best of our knowledge this result is new in the general setting of any subLaplacian on any noncompact Lie group, especially for the endpoint results.

We point out that the problems considered in this paper, namely the algebra property of Sobolev spaces and the $L^{p}$ boundedness of local and global Riesz transforms, have been intensively studied also in the context of Riemannian manifolds. Without any pretense of exhaustiveness, we refer the reader to [4, 5, 10, 12, 33, 37, 43] and the references therein for the study of the boundedness of Riesz transforms and to [6, 9, 15] for Sobolev algebras on Riemannian manifolds satisfying suitable geometric assumptions.

Finally, we mention that the Sobolev algebra property is of great importance in the study of the well-posedness of Cauchy problems involving the operator $\Delta$ in some nonlinear differential equation, such as a nonlinear heat equation, or a nonlinear Schrödinger equation, see [6, 45, 11].

The paper is organized as follows. In Section 2 we recall all preliminaries and notation on nonunimodular Lie groups, the properties of the maximal functions, the estimates of the heat kernel associated with $\Delta$ and the definition of the Hardy and BMO spaces that will be used in the paper. Section 3 is devoted to the study of the boundedness of local Riesz transforms of any order associated with $\Delta$, and we also prove that the analogue of Strichartz and Bohnke [44] and [10] results cited earlier cannot hold in a generic nonunimodular Lie group. In Section 4 we prove two representation formulas for the Sobolev norms in the case when $\alpha$ is in $(0,1)$. Section 5 is devoted to the proof of Theorem 1.2, while we collect in Section 6 some final comments and a discussion on the future developments of this work.

Given two non-negative quantities $A$ and $B$, we write $A \lesssim B$ to indicate that there is $C>0$ such that $A \leq C B$, and the constant $C$ does not depend on the relevant parameters involved in $A$ and $B$. We also write $A \approx B$ when $A \lesssim B$ and $B \lesssim A$.

We wish to thank the anonimous referee for her/his careful reading of the manuscript and for making several useful comments. 


\section{Preliminaries}

The Carnot-Carathéodory metric on $G$ associated with $\mathbf{X}$ is defined as follows. An absolutely continuous curve $\gamma:[0,1] \rightarrow G$ is called horizontal if $\gamma^{\prime}(t)=\sum_{j=1}^{q} a_{j} X_{j}(\gamma(t))$ for

every $t \in[0,1]$. The length of such a curve is defined as $\ell(\gamma)=\int_{0}^{1}\left(\sum_{j=1}^{q}\left|a_{j}\right|^{2}\right)^{1 / 2} \mathrm{~d} t$. The distance of two points $x, y \in G$ is defined as the infimum of the lengths of all horizontal curves joining $x$ to $y$ and denoted by $d_{C}(x, y)$. Since the vector fields $\left\{X_{j}\right\}_{j=1}^{q}$ are left invariant, the metric $d_{C}$ is left invariant. We denote by $|x|$ the distance of a point $x \in G$ from the identity $e$ of $G$ in such metric. For every $x_{0} \in G$ and $r>0$, the open ball centred at $x_{0}$ of radius $r$ is $B\left(x_{0}, r\right)=\left\{x \in G: d_{C}\left(x, x_{0}\right)<r\right\}$. When $x_{0}=e$, we simply write $B_{r}=B(e, r)$, and set

$$
V(r)=\rho\left(B_{r}\right)=\lambda\left(B_{r}\right) .
$$

Notice that for every $x_{0} \in G$ and $r>0$

$$
\rho\left(B\left(x_{0}, r\right)\right)=\delta^{-1}\left(x_{0}\right) V(r) .
$$

It is known [24, 49, 50] that there exists a positive constant $d$ such that

$$
V(r) \approx r^{d} \quad \forall r \in(0,1],
$$

and there exists $D>0$ such that

$$
V(r) \lesssim e^{D r} \quad \forall r \in(1, \infty) .
$$

Notice in particular that the space $\left(G, d_{C}, \rho\right)$ is locally doubling. Moreover, there exists a constant $Q>0$ such that

$$
\int_{B_{r}} \delta^{1 / 2} \mathrm{~d} \rho \lesssim r^{d} e^{Q r} \quad \forall r>0 .
$$

Indeed, when $r \in(0,1]$

$$
\int_{B_{r}} \delta^{1 / 2} \mathrm{~d} \rho \lesssim\left\|\delta^{1 / 2}\right\|_{L^{\infty}\left(B_{1}\right)} V(r) \lesssim r^{d}
$$

when $r \in(1, \infty)$

$$
\int_{B_{r}} \delta^{1 / 2} \mathrm{~d} \rho \lesssim\left(\sup _{B_{r}} \delta\right)^{1 / 2} V(r) \lesssim e^{A r} e^{D r},
$$

where $D$ is the constant in (2.4) and $A=\frac{1}{2}\left(\sum_{i=1}^{q}\left|\left(X_{i} \delta\right)(e)\right|^{2}\right)^{1 / 2}$ (see [25, Proposition 5.7 (ii)]) that gives (2.5). Let us mention that the fact that the integral of $\delta^{1 / 2}$ on any ball grows at most exponentially with respect to the radius of the ball is crucial in the proof of our results.

In the sequel we shall often deal with left invariant operators on $G$ and their kernels. Recall that by the Schwartz kernel theorem, all bounded operators $T: C_{c}^{\infty}(G) \rightarrow \mathcal{D}^{\prime}(G)$ have an integral kernel $K_{T} \in \mathcal{D}^{\prime}(G \times G)$, such that

$$
T f(x)=\int_{G} K_{T}(x, y) f(y) \mathrm{d} \rho(y)
$$


in the sense of distributions. Further, if $T$ is left invariant, then it admits a convolution kernel $k_{T} \in \mathcal{D}^{\prime}(G)$, such that

$$
T f(x)=f * k_{T}(x)=\int_{G} f\left(x y^{-1}\right) k_{T}(y) \mathrm{d} \rho(y) ;
$$

in this case the convolution kernel $k_{T}$ is related to the integral kernel $K_{T}$ by

$$
K_{T}(x, y)=k_{T}\left(y^{-1} x\right) \delta(y) .
$$

We shall list below some notation and well-known results which will be used in the sequel.

2.1. Heat kernel estimates. Let $p_{t}$ be the heat kernel of $\Delta$ at time $t$, i.e. the convolution kernel $k_{T_{t}}$ of the operator $T_{t}=e^{-t \Delta}$ and let $P_{t}$ be the corresponding integral kernel. By [51, Section IX] there exist positive constants $c_{1} \ldots, c_{4}$ such that for every $x \in G$ and $t \in(0,1)$ :

(i) $\int_{G} p_{t} \mathrm{~d} \rho=1$

(ii) $\delta^{1 / 2}(x) V(\sqrt{t})^{-1} e^{-c_{1}|x|^{2} / t} \lesssim p_{t}(x) \lesssim \delta^{1 / 2}(x) V(\sqrt{t})^{-1} e^{-c_{2}|x|^{2} / t}$;

(iii) $\left|\frac{\partial p_{t}}{\partial t}(x)\right| \lesssim \delta^{1 / 2}(x) t^{-1} V(\sqrt{t})^{-1} e^{-c_{3}|x|^{2} / t}$;

(iv) $\left|X_{i} p_{t}(x)\right| \lesssim \delta^{1 / 2}(x) t^{-1 / 2} V(\sqrt{t})^{-1} e^{-c_{4}|x|^{2} / t}$.

By [17, p. 132] there exist $\omega \geq 0, b>0$ such that for every multiindex $J \in\{1, \ldots, q\}^{m}$ and every $t>0$

$$
\left|X_{J} p_{t}(x)\right| \lesssim t^{-\frac{d+m}{2}} e^{\omega t} e^{-b|x|^{2} / t} \quad \forall x \in G .
$$

By using the heat semigroup $T_{t}$ for every $\beta>0$ we define the $g$-function

$$
g_{\beta} f=\left(\int_{0}^{\infty}\left|(t \Delta)^{\beta} T_{t} f\right|^{2} \frac{\mathrm{d} t}{t}\right)^{1 / 2} .
$$

Since $T_{t}$ is a diffusion semigroup symmetric with respect to the measure $\rho$, it is well known that for every $p \in(1, \infty)$ and every $f \in L^{p}$

$$
\|f\|_{p} \approx\left\|g_{\beta} f\right\|_{p} .
$$

See [34, 41].

2.2. Maximal functions. For every $R>0$ we define $\mathcal{B}_{R}$ as the set of all balls of radius $\leq R$. The corresponding local Hardy-Littlewood maximal function with respect to the right Haar measure is given by

$$
M^{R} f(x)=\sup _{B \in \mathcal{B}_{R}, x \in B} \frac{1}{\rho(B)} \int_{B}|f| \mathrm{d} \rho .
$$

The operator $M^{R}$ is bounded on $L^{p}$ for every $p \in(1, \infty]$ and it is of weak type $(1,1)$. We also introduce the modified local Hardy-Littlewood maximal function, with parameter $\beta \in[0,1)$ with respect to the right Haar measure, given by

$$
M_{\beta}^{R} f(x)=\sup _{B \in \mathcal{B}_{R}, x \in B} \frac{1}{\rho(B)^{1-\beta}} \int_{B}|f| \mathrm{d} \rho .
$$

It is easy to show that $M_{\beta}^{R}$ is bounded from $L^{\frac{1}{\beta}}$ to $L^{\infty}$ and from $L^{1}$ to the Lorentz space $L^{\frac{1}{1-\beta}}, \infty$, so that by interpolation it is bounded from $L^{p}$ to $L^{q}$ whenever $\frac{1}{q}=\frac{1}{p}-\beta$ and $p \in\left(1, \frac{1}{\beta}\right]$. 
We denote by $\mathcal{M}_{0}$ the local heat maximal function defined by

$$
\mathcal{M}_{0} f=\sup _{0<t \leq 1}\left|f * p_{t}\right|
$$

It is known that $\mathcal{M}_{0}$ is bounded on $L^{p}$ for $p \in(1, \infty)$ [16, 41].

It is easy to see that the statements of [15, Propositions 7-8-9-10] which concern global maximal operators can be reformulated for the local maximal functions $\mathcal{M}_{0}$ and $M^{R}$ : indeed only the $L^{p}$-boundedness for $p \in(1, \infty)$ of the local maximal functions and the local doubling property are neeeded to adapt the proofs of [15, Propositions 7-8-9-10] to our setting.

2.3. The spaces $\mathfrak{h}^{1}(\rho)$ and $\mathfrak{b m o}(\rho)$. The theory of Hardy spaces of Goldberg type developed in [35] applies to the space $\left(G, d_{C}, \rho\right)$. For the reader's convenience, we recall here briefly the definition of the atomic Hardy space $\mathfrak{h}^{1}(\rho)$ and its dual $\mathfrak{b m o}(\rho)$ and a few related results. We refer the reader to 22, for details on the theory of Goldberg Hardy spaces in the Euclidean setting and to [35, 46] for the corresponding theory in the context of metric spaces and Riemannian manifolds.

Definition 2.1. A standard atom at scale 1 is a function $a \in L^{1}$ supported in a ball $B \in \mathcal{B}_{1}$ such that

(i) $\|a\|_{2} \leq \rho(B)^{-1 / 2}$;

(ii) $\int a \mathrm{~d} \rho=0$.

A global atom at scale 1 is a function $a \in L^{1}$ supported in a ball $B$ of radius exactly 1 such that $\|a\|_{2} \leq \rho(B)^{-1 / 2}$. Standard and global atoms at scale 1 will be referred to as atoms at scale 1 .

The Hardy space $\mathfrak{h}^{1}(\rho)$ is defined as the space

$$
\mathfrak{h}^{1}(\rho)=\left\{f \in L^{1}(\rho): f=\sum_{k} c_{k} a_{k}, a_{k} \text { atom at scale } 1, c_{k} \in \mathbb{C}, \sum_{k}\left|c_{k}\right|<\infty\right\},
$$

endowed with the usual atomic norm

$$
\|f\|_{\mathfrak{h}^{1}}=\inf \left\{\sum_{k}\left|c_{k}\right|: f=\sum_{k} c_{k} a_{k}, a_{k} \text { atom at scale } 1, c_{k} \in \mathbb{C}\right\} .
$$

By [35, Theorem 2] the dual of $\mathfrak{h}^{1}(\rho)$ can be identified with the space $\mathfrak{b} \mathfrak{m o}(\rho)$ of all equivalence classes of locally integrable functions $g$ modulo constants such that

$$
\begin{aligned}
\|g\|_{\mathfrak{b m o}}:= & \sup _{B \in \mathcal{B}_{1}}\left(\frac{1}{\rho(B)} \int_{B}\left|g-g_{B}\right|^{2} \mathrm{~d} \rho\right)^{1 / 2} \\
& +\sup _{x \in G}\left(\frac{1}{\rho(B(x, 1))} \int_{B(x, 1)}|g|^{2} \mathrm{~d} \rho\right)^{1 / 2}<\infty,
\end{aligned}
$$

where $g_{B}=\rho(B)^{-1} \int_{B} g \mathrm{~d} \rho$.

By [13, Theorem 8.2] and [14, Proposition 4.5] the following criterion for the boundedness of integral operators on $G$ holds. 
Proposition 2.2. If $T$ is a bounded operator on $L^{2}$ and its integral kernel $K_{T}$ is a locally integrable function away from the diagonal of $G \times G$ such that

$$
\sup _{B \in \mathcal{B}_{1}} \sup _{y, z \in B} \int_{(2 B)^{c}}\left|K_{T}(x, y)-K_{T}(x, z)\right| \mathrm{d} \rho(x)<\infty
$$

and

$$
\sup _{y \in G} \int_{(B(y, 2))^{c}}\left|K_{T}(x, y)\right| \mathrm{d} \rho(x)<\infty,
$$

then $T$ is bounded from $\mathfrak{h}^{1}(\rho)$ to $L^{1}$.

If $T$ is a bounded operator on $L^{2}$ and its integral kernel $K_{T}$ is a locally integrable function off the diagonal of $G \times G$ such that

$$
\sup _{B \in \mathcal{B}_{1}} \sup _{y, z \in B} \int_{(2 B)^{c}}\left|K_{T}(y, x)-K_{T}(z, x)\right| \mathrm{d} \rho(x)<\infty
$$

and

$$
\sup _{y \in G} \int_{(B(y, 2))^{c}}\left|K_{T}(y, x)\right| \mathrm{d} \rho(x)<\infty,
$$

then $T$ is bounded from $L^{\infty}$ to $\mathfrak{b m o}(\rho)$.

Furthermore, by [35, Theorem 5], the following interpolation result holds, where $(V, W)_{[\theta]}$ denotes the lower complex interpolation space of parameter $\theta \in(0,1)$ between the Banach spaces $V, W$ (see [8]).

Theorem 2.3. Let $\theta \in(0,1)$ and set $p_{\theta}=2 /(2-\theta)$. Then $\left(\mathfrak{h}^{1}(\rho), L^{2}\right)_{[\theta]}=L^{p_{\theta}}$ and $\left(\mathfrak{b m o}(\rho), L^{2}\right)_{[\theta]}=L^{p_{\theta}^{\prime}}$.

\section{BOUNDEDNESS OF THE LOCAL RiESZ TRANSFORMS}

Recall that for every multiindex $J \in\{1, \ldots, q\}^{m}$ the local Riesz transform of order $m$ is defined by $R_{J}^{c}=X_{J}(c I+\Delta)^{-m / 2}$ (see (1.4) ).

In order to prove the boundedness of the local Riesz transforms on $L^{p}$, we shall need to apply the following result concerning the derivatives of the heat kernel at small times. The techniques used in the proof of the following lemma follow closely those used in [23, 37].

Lemma 3.1. Let $B=B\left(c_{B}, r_{B}\right)$ be a ball of radius $r_{B} \leq 1, t \in\left[r_{B}^{2}, 1\right]$ and $y, z \in B$. For every $x \in G$ define

$$
q_{t}(x)=P_{t}(x, y)-P_{t}(x, z) .
$$

Then there exist $\gamma \in(0,1), c_{5}>0$ such that

(i) $\left|q_{t}(x)\right| \lesssim \delta^{1 / 2}\left(c_{B} x\right) V(\sqrt{t})^{-1}\left(\frac{d(y, z)}{\sqrt{t}}\right)^{\gamma} e^{-c_{5}\left|c_{B}^{-1} x\right|^{2} / t}$;

(ii) for every $\beta<2 c_{5}$,

$$
\int_{2 r_{B} \leq\left|c_{B}^{-1} x\right| \leq 2}\left|q_{t}(x)\right|^{2} e^{\beta\left|c_{B}^{-1} x\right|^{2} / t} \mathrm{~d} \rho(x) \lesssim \delta\left(c_{B}\right) V(\sqrt{t})^{-1}\left(\frac{d(y, z)}{\sqrt{t}}\right)^{2 \gamma} ;
$$


(iii) for every $\beta<2 c_{5}$ and $J \in\{1, \ldots, q\}^{m}, m$ a non-negative integer,

$$
\int_{2 r_{B} \leq\left|c_{B}^{-1} x\right| \leq 2}\left|X_{J} q_{t}(x)\right|^{2} e^{\beta\left|c_{B}^{-1} x\right|^{2} / t} \mathrm{~d} \rho(x) \lesssim t^{-m} \delta\left(c_{B}\right) V(\sqrt{t})^{-1}\left(\frac{d(y, z)}{\sqrt{t}}\right)^{2 \gamma} .
$$

Proof. We first prove (i). Fix $x \in G$ and define $u(t, \cdot)=P_{t}(x, \cdot)$. Then $u$ is a solution of the heat equation $\left(\partial_{t}+\Delta\right) u=0$. By applying [37, Proposition 10] (see also [38, Proposition $3.2])$ we deduce that there exists $\gamma \in(0,1)$ such that

$$
\begin{aligned}
\left|q_{t}(x)\right| & \lesssim\left(\frac{d(y, z)}{r_{B}}\right)^{\gamma} \sup _{(\tau, w) \in Q} P_{\tau}(x, w) \\
& \lesssim\left(\frac{d(y, z)}{\sqrt{t}}\right)^{\gamma} \sup _{(\tau, w) \in Q} \delta(w) \delta^{1 / 2}\left(w^{-1} x\right) V(\sqrt{\tau})^{-1} e^{-c_{2}\left|w^{-1} x\right|^{2} / t}
\end{aligned}
$$

where $Q=\left(\frac{4}{9} t, \frac{20}{9} t\right) \times B\left(y, \frac{4}{3} \sqrt{t}\right)$. Using the fact that $y, z \in B, r_{B} \leq 1$ and $r_{B}^{2} \leq t$, we deduce that there exists $c_{5}>0$ such that

$$
\left|q_{t}(x)\right| \lesssim\left(\frac{d(y, z)}{\sqrt{t}}\right)^{\gamma} \delta^{1 / 2}\left(c_{B} x\right) V(\sqrt{t})^{-1} e^{-c_{5}\left|c_{B}^{-1} x\right|^{2} / t} \quad \forall x \in G .
$$

To prove (ii) we apply (i) and the change of variables $c_{B}^{-1} x=v$ to obtain

$$
\begin{aligned}
& \int_{2 r_{B} \leq\left|c_{B}^{-1} x\right| \leq 2}\left|q_{t}(x)\right|^{2} e^{\beta\left|c_{B}^{-1} x\right|^{2} / t} \mathrm{~d} \rho(x) \\
& \quad \lesssim\left(\frac{d(y, z)}{\sqrt{t}}\right)^{2 \gamma} V(\sqrt{t})^{-2} \int_{2 r_{B} \leq\left|c_{B}^{-1} x\right| \leq 2} \delta\left(c_{B} x\right) e^{\left(-2 c_{5}+\beta\right)\left|c_{B}^{-1} x\right|^{2} / t} \mathrm{~d} \rho(x) \\
& \quad \lesssim\left(\frac{d(y, z)}{\sqrt{t}}\right)^{2 \gamma} V(\sqrt{t})^{-2} \delta\left(c_{B}\right) \int_{2 r_{B} \leq|v| \leq 2} e^{\left(-2 c_{5}+\beta\right)|v|^{2} / t} \mathrm{~d} \lambda(v) .
\end{aligned}
$$

If $2 r_{B} \leq \sqrt{t}$ we choose $j_{0}$ as the smallest integer such that $2^{j_{0}+1} \sqrt{t} \geq 2$ and obtain

$$
\begin{aligned}
& \int_{2 r_{B} \leq\left|c_{B}^{-1} x\right| \leq 2}\left|q_{t}(x)\right|^{2} e^{\beta\left|c_{B}^{-1} x\right|^{2} / t} \mathrm{~d} \rho(x) \\
& \lesssim\left(\frac{d(y, z)}{\sqrt{t}}\right)^{2 \gamma} V(\sqrt{t})^{-2} \delta\left(c_{B}\right) \\
& \quad \times\left[\int_{2 r_{B} \leq|v| \leq \sqrt{t}} \mathrm{~d} \lambda(v)+\sum_{j=0}^{j_{0}} \int_{2^{j} \sqrt{t} \leq|v| \leq 2^{j+1} \sqrt{t}} e^{\left(-2 c_{5}+\beta\right) 2^{2 j}} \mathrm{~d} \lambda(v)\right] \\
& \lesssim\left(\frac{d(y, z)}{\sqrt{t}}\right)^{2 \gamma} V(\sqrt{t})^{-2} \delta\left(c_{B}\right)\left[V(\sqrt{t})+\sum_{j=0}^{j_{0}} e^{\left(-2 c_{5}+\beta\right) 2^{2 j}}\left(2^{j+1} \sqrt{t}\right)^{d}\right] \\
& \lesssim\left(\frac{d(y, z)}{\sqrt{t}}\right)^{2 \gamma} V(\sqrt{t})^{-1} \delta\left(c_{B}\right),
\end{aligned}
$$

where we used the fact that $\beta<2 c_{5}$. The proof in the case when $\sqrt{t} \leq 2 r_{B}$ is similar and is omitted. 
We now prove (iii). Take $J \in\{1, \ldots, q\}^{m}$, where $m$ is a non-negative integer. For every $x \in G$, the function $(t, y) \mapsto X_{J, x} P_{t}(x, y)$ is a solution of the heat equation $\partial_{t} u+\Delta u=0$. Thus, by [38, Proposition 3.2]

$$
\left|X_{J} q_{t}(x)\right| \lesssim\left(\frac{d_{C}(y, z)}{\sqrt{t}}\right)^{\gamma} \sup _{(\tau, w) \in Q}\left|X_{J, x} P_{\tau}(x, w)\right|
$$

for some $\gamma \in(0,1)$, where $Q=\left(\frac{4}{9} t, \frac{20}{9} t\right) \times B\left(y, \frac{4}{3} \sqrt{t}\right)$. By means of (2.8) and the assumptions on $r_{B}, t, y, z$ we get

$$
\left|X_{J, x} P_{\tau}(x, w)\right| \lesssim \tau^{-(d+m) / 2} e^{\omega \tau} e^{-b\left|w^{-1} x\right|^{2} / \tau} .
$$

Since $\tau \approx t, t \in(0,1)$ and $t^{-d / 2} \approx V(\sqrt{t})^{-1}$, there exists a constant $c_{5}$ such that

$$
\left|X_{J} q_{t}(x)\right| \lesssim\left(\frac{d_{C}(y, z)}{\sqrt{t}}\right)^{\gamma} t^{-m / 2} V(\sqrt{t})^{-1} e^{-c_{5}\left|c_{B}^{-1} x\right|^{2} / t} .
$$

If $\beta<2 c_{5}$ we can apply (3.1), use the change of variables $c_{B}^{-1} x=v$ and argue as in the proof of (ii) to obtain that

$$
\int_{2 r_{B} \leq\left|c_{B}^{-1} x\right| \leq 2}\left|X_{J} q_{t}(x)\right|^{2} e^{\beta\left|c_{B}^{-1} x\right|^{2} / t} \mathrm{~d} \rho(x) \lesssim t^{-m} \delta\left(c_{B}\right) V(\sqrt{t})^{-1}\left(\frac{d(y, z)}{\sqrt{t}}\right)^{2 \gamma} .
$$

We are now ready to prove Theorem 1.4

Proof of Theorem 1.4. Fix $J \in\{1, \ldots, q\}^{m}$. By [47, Theorem 4.8, IV] if $c>0$ is large enough then the local Riesz transforms $R_{J}^{c}$ is bounded on $L^{2}$. The convolution kernel of $R_{J}^{c}$ is given by

$$
k_{J}^{c}(x)=c_{J} \int_{0}^{1} t^{m / 2-1} e^{-c t} X_{J} p_{t}(x) \mathrm{d} t+c_{J} \int_{1}^{\infty} t^{m / 2-1} e^{-c t} X_{J} p_{t}(x) \mathrm{d} t=k^{0}(x)+k^{\infty}(x) .
$$

We claim that $k^{\infty} \in L^{1}$ if $c$ is sufficiently large. Indeed, we can choose $c$ sufficiently large so that

$$
\left|k^{\infty}(x)\right| \lesssim \int_{1}^{\infty} t^{m / 2-1} e^{-c t} t^{-(d+m) / 2} e^{\omega t} e^{-b|x|^{2} / t} \mathrm{~d} t \lesssim \int_{1}^{\infty} e^{-c^{\prime} t} e^{-b|x|^{2} / t} \mathrm{~d} t,
$$

for some $c^{\prime}>0$. Now define for every $t>0, A_{t}^{i}=B_{2^{i} \sqrt{t}} \backslash B_{2^{i-1} \sqrt{t}}, i \geq 1$. Then by (2.4)

$$
\begin{aligned}
\int_{G}\left|k^{\infty}(x)\right| \mathrm{d} \rho(x) & \lesssim \int_{1}^{\infty} \int_{B(e \sqrt{t})} e^{-c^{\prime} t} e^{-b|x|^{2} / t} \mathrm{~d} \rho(x) \mathrm{d} t+\sum_{i=1}^{\infty} \int_{1}^{\infty} \int_{A_{t}^{i}} e^{-c^{\prime} t} e^{-b|x|^{2} / t} \mathrm{~d} \rho(x) \mathrm{d} t \\
& \lesssim \int_{1}^{\infty} e^{-c^{\prime} t} e^{D \sqrt{t}} \mathrm{~d} t+\sum_{i=1}^{\infty} \int_{1}^{\infty} e^{-c^{\prime} t} e^{-b 2^{2 j}} e^{D 2^{i} \sqrt{t}} \mathrm{~d} t \\
& \lesssim 1+\sum_{i=1}^{\infty} e^{-b 2^{2 i}+\frac{D^{2} 2^{2 i}}{4 c^{\prime}}} \int_{1}^{\infty} e^{-\left(\sqrt{c^{\prime} t}-\frac{D 2^{i}}{2 \sqrt{c}}\right)^{2}} \mathrm{~d} t \\
& \lesssim 1
\end{aligned}
$$

if $c$ is sufficiently large. Thus the convolution operator $f \mapsto f * k^{\infty}$ is bounded on $L^{p}$ for every $p \in[1, \infty]$, and a fortiori it is bounded from $\mathfrak{h}^{1}(\rho)$ to $L^{1}$ and from $L^{\infty}$ to $\mathfrak{b m o}(\rho)$. 
We now consider the kernel $k^{0}$. Choose a smooth cutoff function $\phi$ supported in $B_{1}$ such that $0 \leq \phi \leq 1$. First notice that

$$
\left|(1-\phi(x)) k^{0}(x)\right| \lesssim \int_{0}^{1} t^{m / 2-1} t^{-d / 2-m / 2} e^{-b|x|^{2} / t} \mathrm{~d} t=\int_{|x|^{2}}^{\infty}\left(\frac{|x|^{2}}{u}\right)^{-d / 2} e^{-b u} \mathrm{~d} u \lesssim e^{-b^{\prime}|x|^{2}}
$$

for some $b^{\prime}>0$. Then

$$
\begin{aligned}
\int_{G}\left|(1-\phi(x)) k^{0}(x)\right| \mathrm{d} \rho(x) & \lesssim \sum_{i=1}^{\infty} \int_{A_{1}^{i}} e^{-b^{\prime}|x|} \mathrm{d} \rho(x) \\
& \lesssim \sum_{i=1}^{\infty} e^{-b^{\prime} 2^{2 i}} e^{D 2^{i}} \\
& \lesssim 1
\end{aligned}
$$

Thus the convolution operator $f \mapsto f *(1-\phi) k^{0}$ is bounded on $L^{p}$ for every $p \in[1, \infty]$, and a fortiori it is bounded from $\mathfrak{h}^{1}(\rho)$ to $L^{1}$ and from $L^{\infty}$ to $\mathfrak{b m o}(\rho)$.

It remains to consider the operator $f \mapsto f *\left(\phi k^{0}\right)$ which is bounded on $L^{2}$, as difference of operators bounded on $L^{2}$. Let us denote by $\ell$ the function $\phi k^{0}$ and by $L$ the integral kernel corresponding to the convolution operator with kernel $\ell$, i.e. $L(x, y)=\ell\left(y^{-1} x\right) \delta(y)$. Notice that

$$
|\ell(x)| \lesssim \int_{0}^{1} t^{m / 2-1} t^{-d / 2-m / 2} e^{-b|x|^{2} / t} \mathrm{~d} t=\int_{|x|^{2}}^{\infty}\left(\frac{|x|^{2}}{u}\right)^{-d / 2} e^{-b u} \mathrm{~d} u \lesssim|x|^{-d}
$$

and, for every $j=1, \ldots, q$,

$$
\begin{aligned}
\left|X_{j} \ell(x)\right| & \lesssim\left|k^{0}(x)\right|+\int_{0}^{1} t^{m / 2-1} t^{-d / 2-m / 2-1 / 2} e^{-b|x|^{2} / t} \mathrm{~d} t \\
& \lesssim|x|^{-d}+|x|^{-d-1} \\
& \lesssim|x|^{-d-1} .
\end{aligned}
$$

Notice that since $\ell$ is supported in $B_{1}$ for every ball $B$ of radius 1 and every $y \in B$

$$
\int_{(2 B)^{c}}|L(y, x)| \mathrm{d} \rho(x)=\int_{(2 B)^{c}}|L(x, y)| \mathrm{d} \rho(x)=0 .
$$


Take now a ball $B=B\left(c_{B}, r_{B}\right)$ of radius $r_{B} \leq 1$. For every $y, z \in B$ we have

$$
\begin{aligned}
\int_{(2 B)^{c}}|L(y, x)-L(z, x)| \mathrm{d} \rho(x) & =\int_{(2 B)^{c}}\left|\ell\left(x^{-1} y\right) \delta(x)-\ell\left(x^{-1} z\right) \delta(x)\right| \mathrm{d} \rho(x) \\
& =\int_{\left\{x: d\left(x^{-1}, c_{B}\right)>2 r_{B}\right.}|\ell(x y)-\ell(x z)| \mathrm{d} \rho(x) \\
& =\int_{|u|>2 r_{B}}\left|\ell\left(u c_{B}^{-1} y\right)-\ell\left(u c_{B}^{-1} z\right)\right| \mathrm{d} \rho(u) \\
& =\int_{2 r_{B}<|u| \leq 2}\left|\ell\left(u c_{B}^{-1} y\right)-\ell\left(u c_{B}^{-1} z\right)\right| \mathrm{d} \rho(u) \\
& \lesssim d\left(c_{B}^{-1} y, c_{B}^{-1} z\right) \int_{2 r_{B} \leq|u| \leq 2} \sum_{j=1}^{q}\left|X_{j} \ell(u)\right| \mathrm{d} \rho(u) \\
& \lesssim r_{B} \int_{2 r_{B} \leq|u| \leq 2}|u|^{-d-1} \mathrm{~d} \rho(u),
\end{aligned}
$$

where we have applied (3.3). Choose $i_{0}$ as the biggest integer such that $2^{i_{0}-1} \leq 2 r_{B}$ and consider the annuli $A^{i}=B_{2^{i}} \backslash B_{2^{i-1}}$, with $i_{0} \leq i \leq 1$. By (2.3) we obtain

$$
\begin{aligned}
\int_{(2 B)^{c}}|L(y, x)-L(z, x)| \mathrm{d} \rho(x) & \lesssim r_{B} \sum_{i=i_{0}}^{1} \int_{A^{i}}|u|^{-d-1} \mathrm{~d} \rho(u) \\
& \lesssim r_{B} \sum_{i=i_{0}}^{1} 2^{-i(d+1)} 2^{i d} \\
& \lesssim r_{B} 2^{-i_{0}} \\
& \lesssim 1
\end{aligned}
$$

By Propostion 2.2 the conditions (3.4) and (3.5) imply that $R_{J}^{c}$ is bounded from $L^{\infty}$ to $\mathfrak{b m o}(\rho)$. By Theorem 2.3 we deduce that $R_{J}^{c}$ is bounded on $L^{p}$ for every $p \in[2, \infty)$.

We now prove that $f \mapsto f * k^{0}$ is bounded from $\mathfrak{h}^{1}(\rho)$ to $L^{1}$. We denote by $K^{0}(x, y)$ the corresponding integral kernel. For every ball $B$ of radius 1 and every $y \in B$ by (3.2) we have

$$
\int_{(2 B)^{c}}\left|K^{0}(x, y)\right| \mathrm{d} \rho(x)=\int_{d\left(c_{B}, x\right) \geq 2} \delta(y)\left|k^{0}\left(y^{-1} x\right)\right| \mathrm{d} \rho(x) \leq \int_{|v| \geq 1}\left|k^{0}(v)\right| \mathrm{d} \rho(v) \lesssim 1 .
$$

Take now a ball $B=B\left(c_{B}, r_{B}\right)$ of radius $r_{B} \leq 1$. For every $y, z \in B$ we have

$$
\begin{aligned}
\int_{(2 B)^{c}}\left|K^{0}(x, y)-K^{0}(x, z)\right| \mathrm{d} \rho(x)= & \int_{2 r_{B} \leq d\left(x, c_{B}\right) \leq 2}\left|K^{0}(x, y)-K^{0}(x, z)\right| \mathrm{d} \rho(x) \\
& +\int_{d\left(x, c_{B}\right)>2}\left|K^{0}(x, y)-K^{0}(x, z)\right| \mathrm{d} \rho(x) \\
= & I+I I .
\end{aligned}
$$


By applying (3.2) we have

$$
\begin{aligned}
I I & \leq \int_{d\left(x, c_{B}\right)>2}\left|K^{0}(x, y)\right| \mathrm{d} \rho(x)+\int_{d\left(x, c_{B}\right)>2}\left|K^{0}(x, z)\right| \mathrm{d} \rho(x) \\
& \leq \delta(y) \int_{d\left(v, y^{-1} c_{B}\right)>2}\left|k^{0}(v)\right| \delta^{-1}(y) \mathrm{d} \rho(v)+\delta(z) \int_{d\left(v, z^{-1} c_{B}\right)>2}\left|k^{0}(v)\right| \delta^{-1}(z) \mathrm{d} \rho(v) \\
& \leq 2 \int_{|v|>1}\left|k^{0}(v)\right| \mathrm{d} \rho(v) \\
& \lesssim 1
\end{aligned}
$$

To estimate the integral $I$ we first decompose it as follows:

$$
\begin{aligned}
I \leq & \int_{0}^{1} t^{m / 2-1} e^{-c t} \int_{2 r_{B} \leq d\left(x, c_{B}\right) \leq 2}\left|X_{J, x} P_{t}(x, y)-X_{J, x} P_{t}(x, z)\right| \mathrm{d} \rho(x) \\
= & \int_{0}^{r_{B}^{2}} t^{m / 2-1} e^{-c t} \int_{2 r_{B} \leq d\left(x, c_{B}\right) \leq 2}\left|X_{J, x} P_{t}(x, y)-X_{J, x} P_{t}(x, z)\right| \mathrm{d} \rho(x) \\
& \quad+\int_{r_{B}^{2}}^{1} t^{m / 2-1} e^{-c t} \int_{2 r_{B} \leq d\left(x, c_{B}\right) \leq 2}\left|X_{J, x} P_{t}(x, y)-X_{J, x} P_{t}(x, z)\right| \mathrm{d} \rho(x) \\
= & I_{1}+I_{2}
\end{aligned}
$$

Since $P_{t}(x, y)=\delta(y) p_{t}\left(y^{-1} x\right)$, we have $X_{J, x} P_{t}(x, y)=\delta(y)\left(X^{J} p_{t}\right)\left(y^{-1} x\right)$, so that by (2.8)

$$
\left|X_{J, x} P_{t}(x, y)\right| \lesssim \delta(y) t^{-d / 2-m / 2} e^{\omega t} e^{-b\left|y^{-1} x\right|^{2} / t} .
$$

We then have

$$
\begin{aligned}
I_{1} & \lesssim \int_{0}^{r_{B}^{2}} t^{-1-d / 2} \int_{2 r_{B} \leq\left|c_{B}^{-1} x\right| \leq 2} \delta(y) e^{-b\left|y^{-1} x\right|^{2} / t} \mathrm{~d} \rho(x) \\
& \lesssim \int_{0}^{r_{B}^{2}} t^{-1-d / 2} \delta\left(c_{B}\right) \int_{2 r_{B} \leq|v| \leq 2} e^{-b|v|^{2} / t} \delta^{-1}\left(c_{B} v\right) \mathrm{d} \lambda(v) \\
& \lesssim \int_{2 r_{B} \leq|v| \leq 2} \int_{|v|^{2} / r_{B}^{2}}^{\infty} e^{-b s}\left(\frac{s}{|v|^{2}}\right)^{1+d / 2} \frac{|v|^{2}}{s^{2}} \mathrm{~d} s \mathrm{~d} \rho(v) \\
& \lesssim \int_{2 r_{B} \leq|v| \leq 2}|v|^{-d} \int_{|v|^{2} / r_{B}^{2}}^{\infty} e^{-b^{\prime} s} \mathrm{~d} s \mathrm{~d} \rho(v) \\
& \lesssim \int_{2 r_{B} \leq|v| \leq 2}|v|^{-d} e^{-b^{\prime} \frac{|v|^{2}}{r_{B}^{2}}} \mathrm{~d} \rho(v) .
\end{aligned}
$$

To estimate the last integral we split the domain of integration as the union $\cup_{j=j_{0}}^{1} A_{j}$, where $A_{j}=B_{2^{j}} \backslash B_{2^{j-1}}$, where $j_{0}$ is the largest integer such that $2^{j_{0}-1} \leq 2 r_{B}$ and obtain

$$
\int_{2 r_{B} \leq|v| \leq 2}|v|^{-d} e^{-b^{\prime} \frac{|v|^{2}}{r_{B}^{2}}} \mathrm{~d} \rho(v)=\sum_{j=j_{0}}^{1} 2^{-d j} e^{-b^{\prime} \frac{2^{2 j}}{r_{B}^{2}}} 2^{d j} \lesssim 1 .
$$


It remains to estimate the integral

$$
I_{2}=\int_{r_{B}^{2}}^{1} t^{m / 2-1} e^{-c t} \int_{2 r_{B} \leq\left|c_{B}^{-1} x\right| \leq 2}\left|X_{J} q_{t}(x)\right| \mathrm{d} \rho(x) \mathrm{d} t,
$$

where $q_{t}(x)=P_{t}(x, y)-P_{t}(x, z)$. By applying the Cauchy-Schwarz inequality

$$
\begin{aligned}
\int_{2 r_{B} \leq\left|c_{B}^{-1} x\right| \leq 2}\left|X_{J} q_{t}(x)\right| \mathrm{d} \rho(x) \leq & \left(\int_{2 r_{B} \leq\left|c_{B}^{-1} x\right| \leq 2}\left|X_{J} q_{t}(x)\right|^{2} e^{\beta\left|c_{B}^{-1} x\right|^{2} / t} \mathrm{~d} \rho(x)\right)^{1 / 2} \\
& \times\left(\int_{2 r_{B} \leq\left|c_{B}^{-1} x\right| \leq 2} e^{-\beta\left|c_{B}^{-1} x\right|^{2} / t} \mathrm{~d} \rho(x)\right)^{1 / 2} \\
= & A_{t} \cdot B_{t},
\end{aligned}
$$

with a constant $\beta$ such that $0<\beta<2 c_{5}$, where $c_{5}$ is the constant which appears in Lemma 3.1 ,

To estimate $B_{t}$, when $2 r_{B} \leq \sqrt{t}$ we choose $j_{0}$ as the smallest integer such that $2^{j_{0}+1} \sqrt{t} \geq 2$ and write

$$
\begin{aligned}
\left(B_{t}\right)^{2} & \leq \delta^{-1}\left(c_{B}\right) \int_{2 r_{B} \leq|v| \leq 2} e^{-\beta|v|^{2} / t} \mathrm{~d} \lambda \\
& =\delta^{-1}\left(c_{B}\right)\left(\int_{2 r_{B} \leq|v| \leq \sqrt{t}} e^{-\beta|v|^{2} / t} \mathrm{~d} \lambda+\sum_{j=1}^{j_{0}} \int_{2^{j} \sqrt{t} \leq|v| \leq 2^{j+1} \sqrt{t}} e^{-\beta|v|^{2} / t} \mathrm{~d} \lambda\right) \\
& \lesssim \delta^{-1}\left(c_{B}\right)\left(V(\sqrt{t}) e^{-\beta r_{B}^{2} / t}+\sum_{j=1}^{j_{0}}\left(2^{j} \sqrt{t}\right)^{d} e^{-\beta 2^{j}}\right) \\
& \lesssim \delta^{-1}\left(c_{B}\right) V(\sqrt{t}) e^{-\beta r_{B}^{2} / t} .
\end{aligned}
$$

When $\sqrt{t} \leq 2 r_{B}$ we argue in a similar way and obtain $\left(B_{t}\right)^{2} \lesssim \delta^{-1}\left(c_{B}\right) V(\sqrt{t}) e^{-\beta r_{B}^{2} / t}$. By the previous estimate and Lemma 3.1 we get

$$
\begin{aligned}
\int_{2 r_{B} \leq\left|c_{B}^{-1} x\right| \leq 2}\left|X_{J} q_{t}(x)\right| & \mathrm{d} \rho(x) \\
& \lesssim \delta\left(c_{B}\right)^{1 / 2} t^{-m / 2} V(\sqrt{t})^{-1 / 2} \delta^{-1 / 2}\left(c_{B}\right) V(\sqrt{t})^{1 / 2} e^{-\beta r_{B}^{2} / 2 t}\left(\frac{r_{B}}{\sqrt{t}}\right)^{\gamma},
\end{aligned}
$$

so that

$$
I_{2} \lesssim \int_{r_{B}^{2}}^{1} t^{m / 2-1} e^{-c t} t^{-m / 2} e^{-\beta r_{B}^{2} / 2 t}\left(\frac{r_{B}}{\sqrt{t}}\right)^{\gamma} \mathrm{d} t \lesssim 1 .
$$

This shows that for every ball $B$ of radius $r_{B} \leq 1$ and every points $y, z \in B$

$$
\int_{(2 B)^{c}}\left|K^{0}(x, y)-K^{0}(x, z)\right| \mathrm{d} \rho(x) \lesssim 1
$$

which together with condition (3.6) implies that the integral operator with integral kernel $K^{0}$ is bounded from $\mathfrak{h}^{1}(\rho)$ to $L^{1}$. By Theorem 2.3 we deduce that $R_{J}^{c}$ is bounded on $L^{p}$ for every $p \in(1,2]$.

As a corollary of Theorem 1.4 we have the following result involving Sobolev spaces.

Corollary 3.2. For every $p \in(1, \infty)$ the following hold: 
(i) if $\ell \in \mathbb{N}$, then $f \in L_{\ell}^{p}$ if and only if $X_{J} f \in L^{p}$ for every $J \in\{1, \ldots, q\}^{m}$ with $m \leq \ell$ and

$$
\|f\|_{\ell, p} \approx \sum_{J \in\{1, \ldots, q\}^{m}, m \leq \ell}\left\|X_{J} f\right\|_{p} .
$$

(ii) for every $\alpha \geq 0, f \in L_{\alpha+1}^{p}$ if and only if $f \in L_{\alpha}^{p}$ and $X_{i} f \in L_{\alpha}^{p}, i=1, \ldots, q$, and

$$
\|f\|_{\alpha+1, p} \approx\|f\|_{\alpha, p}+\sum_{i=1}^{q}\left\|X_{i} f\right\|_{\alpha, p} .
$$

(iii) for every $p \in(1, \infty), \alpha \geq 0$ and $c>0$,

$$
\|f\|_{\alpha, p} \approx\left\|(I+\Delta)^{\alpha / 2} f\right\|_{p} \approx\left\|(c I+\Delta)^{\alpha / 2} f\right\|_{p} \quad \forall f \in L_{\alpha}^{p} .
$$

Proof. Statement (i) follows from the $L^{p}$-boundedness of local Riesz transforms $R_{J}^{c}$ arguing as in [36, Theorem 5.14]. Statement (ii) has the same proof as [15, Proposition 19].

To prove (iii) we observe that by [28, Theorem 6.4] $L_{\alpha}^{p}=\operatorname{dom}\left((I+\Delta)^{\alpha / 2}\right)$, and then arguing as in [19, Propositions 3.16, 4.1], the equivalence of norms on the left follows. The one on the right now follows at once.

We are now in the position to show that on a general nonunimodular group $G$, the property that $L_{\alpha}^{p}(G)$ is an algebra when the product $\alpha p$ is sufficiently large, cannot hold true. We recall that when $G$ is a nilpotent Lie group of homogeneuous dimension $Q$, and $\Delta$ is a subLaplacian, then $L_{\alpha}^{p}(G)$ is an algebra provide $\alpha p>Q$, see [10] and the earlier paper 44 for the case of $\mathbb{R}^{n}$. The counterexample appears in the case of the "ax $+b$-group". Precisely, let $G=\mathbb{R} \ltimes \mathbb{R}_{+}$, with product given by $(x, a)\left(x^{\prime}, a^{\prime}\right)=\left(x+a x^{\prime}, a a^{\prime}\right)$. Then, the right Haar measure is $\mathrm{d} \rho(a, x)=a^{-1} \mathrm{~d} a \mathrm{~d} x, \delta(x, a)=a^{-1}$ and a basis for the left invariant vector fields is $\left\{X_{0}, X_{1}\right\}$, where $X_{0}=a \partial_{a}$ and $X_{1}=a \partial_{x}$. Then, we have the following result.

Theorem 3.3. Let $G=\mathbb{R} \ltimes \mathbb{R}_{+}$. Then, for every $p \in(1,+\infty)$ and $k$ positive integer, the Sobolev space $L_{k}^{p}(G)$ is not an algebra.

Proof. Let $\psi$ be a nonnegative function in $C_{c}^{\infty}(0,1)$ such that $\psi=1$ on $[1 / 4,3 / 4]$, and $\chi$ a nonnegative function in $C_{c}^{\infty}(-1,1)$ such that $\chi=1$ on $[0,1 / 2]$. For $\gamma, r>0$ define

$$
g(x, a)=\psi\left(x / a^{r}\right) \chi(a) a^{-\gamma} .
$$

We claim that if $\gamma$ and $r$ satisfy the condition

$$
\frac{r}{2 p}<\gamma<\frac{r}{p}+k_{1}(1-r), \quad \text { for } k_{1}=0,1, \ldots, k,
$$

then $g \in L_{k}^{p}$, but $g^{2} \notin L^{p}$. Note that condition (3.9) is satisfied by any pair $r, \gamma$ with $0<r<1$ and $r /(2 p)<\gamma<r / p$.

By Corollary $3.2(\mathrm{i})$, in order to show that $g \in L_{k}^{p}$, since $\left\{X_{0}, X_{1}\right\}$ is a basis, we need to show that $X_{0}^{k_{0}} X_{1}^{k_{1}} g \in L^{p}$ when $0 \leq k_{0}+k_{1} \leq k$. Using induction, it is easy to check that

- $X_{1}^{k_{1}} \psi\left(x / a^{r}\right)=a^{k_{1}-r k_{1}} \widetilde{\psi}\left(x / a^{r}\right)$ for another $\widetilde{\psi} \in C_{c}^{\infty}(0,1)$;

- if $\widetilde{\psi} \in C_{c}^{\infty}(0,1)$ and $j \in \mathbb{N}$, then $X_{0}^{j} \widetilde{\psi}\left(x / a^{r}\right)=\widetilde{\psi}_{1}\left(x / a^{r}\right)$, for another $\widetilde{\psi}_{1} \in C_{c}^{\infty}(0,1)$;

- $X_{0}^{j} a^{-q}=c a^{-q}$, for some constant $c$, for all $q>0$ and $j \in \mathbb{N}$;

- if any derivative falls on $\chi$, the resulting term is of the form $\Psi \in C_{c}^{\infty}(G)$. 
Therefore, $X_{0}^{k_{0}} X_{1}^{k_{1}} g$ is sum of terms of the form

$$
\widetilde{\psi}\left(x / a^{r}\right) \chi(a) a^{k_{1}-k_{1} r-\gamma}+\widetilde{\Psi}(a, x)
$$

for some $\widetilde{\psi} \in C_{c}^{\infty}(0,1)$ and $\widetilde{\Psi} \in C_{c}^{\infty}(G)$. Thus, $X_{0}^{k_{0}} X_{1}^{k_{1}} g \in L^{p}$ if

$$
\int_{G}\left|\widetilde{\psi}\left(x / a^{r}\right) \chi(a) a^{k_{1}(1-r)-\gamma}\right|^{p} \frac{\mathrm{d} a \mathrm{~d} x}{a} \lesssim \int_{0}^{1} a^{k_{1}(1-r) p-\gamma p+r-1} \mathrm{~d} a<+\infty,
$$

which is the case if and only if $k_{1}(1-r) p-\gamma p+r>0$; which is the inequality on the right of (3.9).

On the other hand,

$$
\left\|g^{2}\right\|_{p}^{p} \approx \int_{0}^{1 / 2} a^{-2 \gamma p+r-1} \mathrm{~d} a
$$

which is infinite if $\gamma>r /(2 p)$.

Remark 3.4. In [50] Varopoulos showed that on a Lie group $G, L_{1}^{p}$ continuously embeds in $L^{q}\left(\delta^{s}\right)$ if $1 \leq p \leq q<\infty$ and $s=1-\frac{p}{q}$. We note that the function $g$ constructed in the theorem is in $L_{1}^{p}$ for $\gamma, r$ satisfying (3.9), while, on the other hand $g \notin L^{\infty}$, as it is easy to check. Thus, this function also shows that the Sobolev embedding theorem cannot hold at the limiting point $q=\infty$ and the modular function $\delta$ appears in a natural way in the Sobolev embeddings when the group is nonunimodular — see also [11].

\section{Sobolev norms in the CASE $\alpha \in(0,1)$}

We shall give two representation formulas for the Sobolev norms when $\alpha \in(0,1)$.

4.1. A representation formula for the Sobolev norm in terms of $S_{\alpha}^{\text {loc }}$. Recall that in (1.2) we have defined the quantity $S_{\alpha}^{\text {loc }} f$. We now prove Theorem 1.3 (i).

Proof of Theorem 1.3 (i). STEP I. We shall prove that

$$
\left\|\Delta^{\alpha / 2} f\right\|_{p} \lesssim\left\|S_{\alpha}^{\text {loc }} f\right\|_{p}+\|f\|_{p} \quad \forall f \in L_{\alpha}^{p} .
$$

We observe that

$$
\begin{aligned}
\left(g_{1-\alpha / 2} \Delta^{\alpha / 2} f(x)\right)^{2} & =\int_{0}^{\infty} t^{1-\alpha}\left|\Delta T_{t} f(x)\right|^{2} \mathrm{~d} t \\
& =\int_{0}^{1} t^{1-\alpha}\left|\Delta T_{t} f(x)\right|^{2} \mathrm{~d} t+\int_{1}^{\infty} t^{1-\alpha}\left|\Delta T_{t} f(x)\right|^{2} \mathrm{~d} t \\
& =:\left(g_{1-\alpha / 2,0} \Delta^{\alpha / 2} f(x)\right)^{2}+\left(g_{1-\alpha / 2, \infty} \Delta^{\alpha / 2} f(x)\right)^{2} .
\end{aligned}
$$

Notice that

$$
\left(g_{1-\alpha / 2, \infty} \Delta^{\alpha / 2} f(x)\right)^{2} \leq \int_{1}^{\infty}\left|t \Delta T_{t} f(x)\right|^{2} \frac{\mathrm{d} t}{t} \leq\left(g_{1} f(x)\right)^{2},
$$

so that by (2.9)

$$
\left\|g_{1-\alpha / 2, \infty} \Delta^{\alpha / 2} f\right\|_{p} \leq\left\|g_{1} f\right\|_{p} \lesssim\|f\|_{p}
$$


To estimate $g_{1-\alpha / 2,0} \Delta^{\alpha / 2} f$ we first notice that for every $t \in(0,1)$ and $x \in G$, since $\frac{\partial}{\partial t} \int_{G} p_{t} \mathrm{~d} \rho=0$, we have

$$
\begin{aligned}
\left|\Delta T_{t} f(x)\right| & =\left|\frac{\partial}{\partial t} T_{t} f(x)\right|=\left|\frac{\partial}{\partial t}\left(\int_{G} f\left(x y^{-1}\right) p_{t}(y) \mathrm{d} \rho(y)-\int_{G} f(x) p_{t}(y) \mathrm{d} \rho(y)\right)\right| \\
& \leq \int_{G}\left|f\left(x y^{-1}\right)-f(x)\right|\left|\frac{\partial p_{t}(y)}{\partial t}\right| \mathrm{d} \rho(y) .
\end{aligned}
$$

Using estimate (iii) in Subsection 2.1 for the derivative of the heat kernel and CauchySchwarz's inequality we have

$$
\begin{aligned}
& \left(g_{1-\alpha / 2,0} \Delta^{\alpha / 2} f(x)\right)^{2} \\
& \lesssim \int_{0}^{1} t^{1-\alpha-2} V(\sqrt{t})^{-2}\left(\int_{|y|<\sqrt{t}}\left|f\left(x y^{-1}\right)-f(x)\right| \delta^{1 / 2}(y) e^{-c_{3}|y|^{2} / t} \mathrm{~d} \rho(y)\right)^{2} \mathrm{~d} t \\
& \quad+\sum_{k=0}^{\infty} \int_{0}^{1} t^{1-\alpha-2} V(\sqrt{t})^{-2}\left(\int_{2^{k}<|y|<2^{k+1} \sqrt{t}}\left|f\left(x y^{-1}\right)-f(x)\right| \delta^{1 / 2}(y) e^{-c_{3}|y|^{2} / t} \mathrm{~d} \rho(y)\right)^{2} \mathrm{~d} t \\
& \lesssim \int_{0}^{1} t^{1-\alpha-2} V(\sqrt{t})^{-2}\left(\int_{|y|<\sqrt{t}}\left|f\left(x y^{-1}\right)-f(x)\right| \mathrm{d} \rho(y)\right)^{2} \mathrm{~d} t \\
& \quad+\sum_{k=0}^{\infty} e^{-c^{\prime} 2^{2 k}} \int_{0}^{1} t^{1-\alpha-2} V(\sqrt{t})^{-2}\left(\int_{|y|<2^{k+1} \sqrt{t}}\left|f\left(x y^{-1}\right)-f(x)\right| \delta^{1 / 2}(y) \mathrm{d} \rho(y)\right)^{2} \mathrm{~d} t .
\end{aligned}
$$

By the change of variables $u=2^{k+1} \sqrt{t}$ we obtain

$$
\begin{aligned}
& \left(g_{1-\alpha / 2,0} \Delta^{\alpha / 2} f(x)\right)^{2} \\
& \lesssim \int_{0}^{1} \frac{1}{u^{1+2 \alpha} V(u)^{2}}\left(\int_{|y|<u}\left|f\left(x y^{-1}\right)-f(x)\right| \mathrm{d} \rho(y)\right)^{2} \mathrm{~d} u \\
& \quad+\sum_{k=0}^{\infty} e^{-c^{\prime} 2^{2 k}} \int_{0}^{2^{k+1}} \frac{1}{\left(2^{-k-1}\right)^{2 \alpha} u^{1+2 \alpha} V\left(2^{-k-1} u\right)^{2}}\left(\int_{|y|<u}\left|f\left(x y^{-1}\right)-f(x)\right| \delta^{1 / 2}(y) \mathrm{d} \rho(y)\right)^{2} \mathrm{~d} u \\
& \lesssim\left(S_{\alpha}^{\operatorname{loc}} f(x)\right)^{2} \\
& \quad+\sum_{k=0}^{\infty} e^{-c^{\prime} 2^{2 k}} \int_{0}^{1} \frac{1}{\left(2^{-k-1}\right)^{2 \alpha} u^{1+2 \alpha} V\left(2^{-k-1} u\right)^{2}}\left(\int_{|y|<u}\left|f\left(x y^{-1}\right)-f(x)\right| \mathrm{d} \rho(y)\right)^{2} \mathrm{~d} u \\
& \quad+\sum_{k=0}^{\infty} e^{-c^{\prime} 2^{2 k}} \int_{1}^{2^{k+1}} \frac{1}{\left(2^{-k-1}\right)^{2 \alpha} u^{1+2 \alpha} V\left(2^{-k-1} u\right)^{2}}\left(\int_{|y|<u}\left|f\left(x y^{-1}\right)-f(x)\right| \delta^{1 / 2}(y) \mathrm{d} \rho(y)\right)^{2} \mathrm{~d} u .
\end{aligned}
$$


By (1.2) and formula (2.3) we obtain that

$$
\begin{aligned}
& \left(g_{1-\alpha / 2,0} \Delta^{\alpha / 2} f(x)\right)^{2} \\
& \lesssim\left(S_{\alpha}^{\text {loc }} f(x)\right)^{2}+\left(S_{\alpha}^{\text {loc }} f(x)\right)^{2} \sum_{k=0}^{\infty} e^{-c^{\prime} 2^{2 k}}\left(2^{k+1}\right)^{2 \alpha+2 d} \\
& \quad+\sum_{k=0}^{\infty} e^{-c^{\prime} 2^{2 k}}\left(2^{k+1}\right)^{2 \alpha+2 d} \int_{1}^{2^{k+1}} \frac{1}{u^{1+2 \alpha+2 d}}|f(x)|^{2}\left(\int_{|y|<u} \delta^{1 / 2}(y) \mathrm{d} \rho(y)\right)^{2} \mathrm{~d} u \\
& \quad+\sum_{k=0}^{\infty} e^{-c^{\prime} 2^{2 k}}\left(2^{k+1}\right)^{2 \alpha+2 d} \int_{1}^{2^{k+1}} \frac{1}{u^{1+2 \alpha+2 d}}\left(\int_{|y|<u}\left|f\left(x y^{-1}\right)\right| \delta^{1 / 2}(y) \mathrm{d} \rho(y)\right)^{2} \mathrm{~d} u \\
& \lesssim\left(S_{\alpha}^{\operatorname{loc}} f(x)\right)^{2}+\sum_{k=0}^{\infty} J_{k}(x)+\sum_{k=0}^{\infty} I_{k}(x) .
\end{aligned}
$$

By (2.5) we deduce that

$$
\begin{aligned}
J_{k}(x) & \lesssim e^{-c^{\prime} 2^{2 k}}\left(2^{k+1}\right)^{2 \alpha+2 d} \int_{1}^{2^{k+1}} \frac{1}{u^{1+2 \alpha+2 d}}|f(x)|^{2} u^{2 d} e^{2 Q u} \mathrm{~d} u \\
& \lesssim|f(x)|^{2} e^{-c^{\prime} 2^{2 k}}\left(2^{k+1}\right)^{2 \alpha+2 d} e^{2 Q 2^{k}},
\end{aligned}
$$

so that $\sum_{k=0}^{\infty} J_{k}(x) \lesssim|f(x)|^{2}$. We now notice that there exists $c^{\prime \prime}>0$ such that

$$
\left\|\left(\sum_{k=0}^{\infty} I_{k}\right)^{1 / 2}\right\|_{p} \lesssim \sum_{k=0}^{\infty} e^{-c^{\prime \prime} 2^{2 k}}\left\|\left(\int_{1}^{2^{k+1}}\left(\int_{|y|<u}\left|f\left(x y^{-1}\right)\right| \delta^{1 / 2}(y) \mathrm{d} \rho(y)\right)^{2}\right)^{1 / 2} \mathrm{~d} u\right\|_{p} .
$$

For every integer $k$, by Minkowski inequality, we get

$$
\begin{aligned}
& \left(\int_{1}^{2^{k+1}}\left(\int_{G}\left|f\left(x y^{-1}\right)\right| \chi_{B_{u}}(y) \delta^{1 / 2}(y) \mathrm{d} \rho(y)\right)^{2} \mathrm{~d} u\right)^{1 / 2} \\
& \lesssim \int_{G}\left(\int_{1}^{2^{k+1}}\left|f\left(x y^{-1}\right)\right|^{2} \chi_{B_{u}}(y) \delta(y) \mathrm{d} u\right)^{1 / 2} \mathrm{~d} \rho(y) \\
& \lesssim \int_{B_{1}}\left|f\left(x y^{-1}\right)\right| \delta^{1 / 2}(y)\left(\int_{1}^{2^{k+1}} \mathrm{~d} u\right)^{1 / 2} \mathrm{~d} \rho(y) \\
& \quad+\int_{1 \leq|y| \leq 2^{k+1}}\left|f\left(x y^{-1}\right)\right| \delta^{1 / 2}(y)\left(\int_{|y|}^{2^{k+1}} \mathrm{~d} u\right)^{1 / 2} \mathrm{~d} \rho(y) \\
& \lesssim \int_{B_{1}}|f(x z)| 2^{k / 2} \mathrm{~d} \lambda(z)+\int_{1 \leq|z| \leq 2^{k+1}}|f(x z)| \delta^{1 / 2}\left(z^{-1}\right) 2^{k / 2} \mathrm{~d} \lambda(z) .
\end{aligned}
$$


We then obtain, by applying once again Minkowski inequality,

$$
\begin{aligned}
& \left\|\left(\int_{1}^{2^{k+1}}\left(\int_{G}\left|f\left(x y^{-1}\right)\right| \chi_{B_{u}}(y) \delta^{1 / 2}(y) \mathrm{d} \rho(y)\right)^{2} \mathrm{~d} u\right)^{1 / 2}\right\|_{p} \\
& \lesssim 2^{k / 2} \int_{B_{1}}\left(\int_{G}|f(x z)|^{p} \mathrm{~d} \rho(x)\right)^{1 / p} \mathrm{~d} \lambda(z)+2^{k / 2} \int_{1 \leq|z| \leq 2^{k+1}}\left(\int_{G}|f(x z)|^{p} \mathrm{~d} \rho(x)\right)^{1 / p} \delta^{-1 / 2}(z) \mathrm{d} \lambda(z) \\
& \lesssim 2^{k / 2}\|f\|_{p}+2^{k / 2}\|f\|_{p} \int_{B_{2^{k+1}}} \delta^{1 / 2} \mathrm{~d} \rho \\
& \lesssim 2^{k / 2+k d}\|f\|_{p} e^{Q 2^{k}}
\end{aligned}
$$

where we have applied (2.5). We then have

$$
\left\|\left(\sum_{k=0}^{\infty} I_{k}\right)^{1 / 2}\right\|_{p} \lesssim \sum_{k=0}^{\infty} e^{-c^{\prime \prime} 2^{2 k}} 2^{k / 2+k d}\|f\|_{p} e^{Q 2^{k}} \lesssim\|f\|_{p}
$$

In conclusion, by (4.2) and (4.3) we get

$$
\left\|g_{1-\alpha / 2,0} \Delta^{\alpha / 2} f\right\|_{p} \lesssim\left\|S_{\alpha}^{\mathrm{loc}} f\right\|_{p}+\|f\|_{p},
$$

as required.

STEP II. We shall prove that

$$
\left\|S_{\alpha}^{\mathrm{loc}} f\right\|_{p} \lesssim\left\|\Delta^{\alpha / 2} f\right\|_{p}+\|f\|_{p} \quad \forall f \in L_{\alpha}^{p} .
$$

To prove it we write $f=\left(f-T_{1} f\right)+T_{1} f$ and we estimate $\left\|S_{\alpha}^{\text {loc }}\left(f-T_{1} f\right)\right\|_{p}$ and $\left\|S_{\alpha}^{\text {loc }} T_{1} f\right\|_{p}$, separately.

Arguing as in [15] we write

$$
f-T_{1} f=\sum_{m=-\infty}^{-1} f_{m}, \text { where } f_{m}=-\int_{2^{m}}^{2^{m+1}} \frac{\partial}{\partial t} T_{t} f \mathrm{~d} t, \quad \text { and } \quad g_{m}=\int_{2^{m-1}}^{2^{m}}\left|\frac{\partial}{\partial t} T_{t} f\right| \mathrm{d} t .
$$

We then obtain

$$
\begin{aligned}
& \left(S_{\alpha}^{\operatorname{loc}}\left(f-T_{1} f\right)(x)\right)^{2} \\
& =\int_{0}^{1} \frac{1}{u^{1+2 \alpha} V(u)^{2}}\left(\int_{|y|<u}\left|\left(f-T_{1} f\right)\left(x y^{-1}\right)-\left(f-T_{1} f\right)(x)\right| \mathrm{d} \rho(y)\right)^{2} \mathrm{~d} u \\
& =\sum_{j=-\infty}^{-1} \int_{2^{j}}^{2^{j+1}} \frac{1}{u^{1+2 \alpha} V(u)^{2}}\left(\int_{|y|<u}\left|\left(f-T_{1} f\right)\left(x y^{-1}\right)-\left(f-T_{1} f\right)(x)\right| \mathrm{d} \rho(y)\right)^{2} \mathrm{~d} u \\
& \lesssim \sum_{j=-\infty}^{-1} \frac{2^{j}}{\left(2^{j}\right)^{1+2 \alpha+2 d}}\left(\int_{|y|<2^{j+1}}\left|\left(f-T_{1} f\right)\left(x y^{-1}\right)-\left(f-T_{1} f\right)(x)\right| \mathrm{d} \rho(y)\right)^{2},
\end{aligned}
$$

where we applied (2.3). Notice that

$$
\begin{aligned}
& \frac{1}{2^{j d}} \int_{|y|<2^{j+1}}\left|\left(f-T_{1} f\right)\left(x y^{-1}\right)-\left(f-T_{1} f\right)(x)\right| \mathrm{d} \rho(y) \\
& \lesssim \sum_{m=-\infty}^{-1} \frac{1}{2^{j d}} \int_{|y|<2^{j+1}}\left|f_{m}\left(x y^{-1}\right)-f_{m}(x)\right| \mathrm{d} \rho(y) .
\end{aligned}
$$


If $m<2 j+3$, then

$$
\frac{1}{2^{j d}} \int_{|y|<2^{j+1}}\left|f_{m}\left(x y^{-1}\right)-f_{m}(x)\right| \mathrm{d} \rho(y) \lesssim M^{1} g_{m+1}(x),
$$

where $M^{1}$ is the local maximal function defined in (2.10). In order to treat the case when $m \geq 2 j+3$, we notice that for every $j \leq-1, y \in B_{2^{j+1}}$ and $x \in G$

$$
\left|f_{m}\left(x y^{-1}\right)-f_{m}(x)\right| \leq 2^{j+1} \sup \left\{\left|X_{i} f_{m}(w)\right|: i=1, \ldots, q,\left|w^{-1} x\right| \leq 2^{j+1}\right\} .
$$

Since

$$
f_{m}=-2 \int_{2^{m-1}}^{2^{m}} \frac{\partial}{\partial t}\left(T_{2 t} f\right) \mathrm{d} t=-4 \int_{2^{m-1}}^{2^{m}} T_{t} \frac{\partial}{\partial t}\left(T_{t} f\right) \mathrm{d} t
$$

by applying the estimates of the heat kernel given in Subsection 2.1, for every $w$ such that $\left|w^{-1} x\right| \leq 2^{j+1}$ we have

$$
\begin{aligned}
\left|X_{i} f_{m}(w)\right| & \lesssim \int_{2^{m-1}}^{2^{m}} \int_{G}\left|\frac{\partial}{\partial t}\left(T_{t} f\right)(z)\right|\left|X_{i} p_{t}\left(z^{-1} w\right)\right| \mathrm{d} \lambda(z) \\
& \lesssim \int_{G} \int_{2^{m-1}}^{2^{m}}\left|\frac{\partial}{\partial t}\left(T_{t} f\right)(z)\right| t^{-1 / 2} V(\sqrt{t})^{-1} \delta^{1 / 2}\left(z^{-1} w\right) e^{-c\left|z^{-1} w\right|^{2} / t} \mathrm{~d} t \mathrm{~d} \lambda(z) \\
& \lesssim 2^{-m / 2} 2^{-m d / 2} \int_{G} g_{m}(z) \delta^{1 / 2}\left(z^{-1} x\right) e^{-c\left|z^{-1} x\right|^{2} / 2^{m}} \mathrm{~d} \lambda(z) \\
& \lesssim 2^{-m / 2} T_{c 2^{m}} g_{m}(x),
\end{aligned}
$$

for a suitable constant $c$. From (4.6) if follows that

$$
\begin{aligned}
\frac{1}{2^{j d}} \int_{|y|<2^{j+1}}\left|f_{m}\left(x y^{-1}\right)-f_{m}(x)\right| \mathrm{d} \rho(y) & \lesssim \frac{1}{2^{j d}} 2^{j+1} 2^{-m / 2} T_{c 2^{m}} g_{m}(x) 2^{j d} \\
& \lesssim 2^{j-m / 2} T_{c 2^{m}} g_{m}(x) .
\end{aligned}
$$

Thus, by (4.5) and (4.7)

$$
\left(S_{\alpha}^{\mathrm{loc}}\left(f-T_{1} f\right)(x)\right)^{2} \lesssim \sum_{j=-\infty}^{-1} \frac{1}{\left(2^{j}\right)^{2 \alpha}}\left(\sum_{m=-\infty}^{2 j+3-1} M^{1} g_{m+1}(x)+\sum_{m=2 j+3}^{-1} 2^{j-m / 2} T_{c 2^{m}} g_{m}(x)\right)^{2} .
$$

We can argue as in [15, p.298-303, 308-309] to deduce that

$$
\left\|S_{\alpha}^{\mathrm{loc}}\left(f-T_{1} f\right)(x)\right\|_{p} \lesssim\left\|\left(\sum_{m=-\infty}^{-1} 2^{-m \alpha} g_{m}^{2}\right)^{1 / 2}\right\|_{p} .
$$

Since

$$
\sum_{m=-\infty}^{-1} 2^{-m \alpha} g_{m}^{2}(x) \lesssim \sum_{m=-\infty}^{-1} \int_{2^{m-1}}^{2^{m}}\left|\frac{\partial}{\partial t} T_{t} f(x)\right|^{2} \mathrm{~d} t \lesssim g_{1-\alpha / 2} \Delta^{\alpha / 2} f(x)^{2},
$$

we have

$$
\left\|S_{\alpha}^{\mathrm{loc}}\left(f-T_{1} f\right)\right\|_{p} \lesssim\left\|g_{1-\alpha / 2} \Delta^{\alpha / 2} f\right\|_{p} \lesssim\left\|\Delta^{\alpha / 2} f\right\|_{p} .
$$

In order to estimate the norm of $S_{\alpha}^{\text {loc }} T_{1} f$ we first notice that for every $x \in G$ and $y \in B_{1}$

$$
\begin{aligned}
\left|T_{1} f\left(x y^{-1}\right)-T_{1} f(x)\right| & \lesssim|y| \sup \left\{\left|X_{i} T_{1} f(w)\right|:\left|w^{-1} x\right| \leq|y|\right\} \\
& \lesssim|y| \sup \left\{\left|X_{i} T_{1} f(w)\right|:\left|w^{-1} x\right| \leq 1\right\} .
\end{aligned}
$$


By the estimates of the heat kernel and its derivatives in Subsection 2.1 there exists $t_{0}>0$ such that for every $w$ such that $\left|w^{-1} x\right| \leq 1$

$$
\begin{aligned}
\left|X_{i} T_{1} f(w)\right| & =\left|f * X_{i} p_{1}(w)\right| \\
& \leq \int\left|f\left(w v^{-1}\right)\right|\left|X_{i} p_{1}(v)\right| \mathrm{d} \rho \\
& \lesssim \int\left|f\left(w v^{-1}\right)\right| \delta^{1 / 2}(v) e^{-c|v|^{2}} \mathrm{~d} \rho(v) \\
& \lesssim \int\left|f\left(w v^{-1}\right)\right| p_{t_{0}}(v) \mathrm{d} \rho(v) \\
& =\int|f(z)| p_{t_{0}}\left(z^{-1} w\right) \mathrm{d} \lambda(z) \\
& \lesssim \int|f(z)| p_{t_{0}}\left(z^{-1} x\right) \mathrm{d} \lambda(z) \\
& =T_{t_{0}}|f|(x) .
\end{aligned}
$$

Thus

$$
\begin{aligned}
S_{\alpha}^{\mathrm{loc}} T_{1} f(x)^{2} & =\int_{0}^{1}\left(\frac{1}{u^{\alpha} V(u)} \int_{|y|<u}\left|T_{1} f\left(x y^{-1}\right)-T_{1} f(x)\right| \mathrm{d} \rho(y)\right)^{2} \frac{\mathrm{d} u}{u} \\
& \lesssim \int_{0}^{1} \frac{1}{u^{2 \alpha} V(u)^{2}}\left(\int_{|y|<u} u T_{t_{0}}|f|(x) \mathrm{d} \rho(y)\right)^{2} \frac{\mathrm{d} u}{u} \\
& \lesssim T_{t_{0}}|f|(x)^{2},
\end{aligned}
$$

where we used the fact that $\alpha \in(0,1)$.

$$
\left\|S_{\alpha}^{\mathrm{loc}} T_{1} f\right\|_{p} \lesssim\left\|T_{t_{0}}|f|\right\|_{p} \lesssim\|f\|_{p}
$$

which together with (4.8) gives (4.4), as required.

The representation formula that we just proved is the key ingredient to show the following lemma, which will be useful to prove the "interpolation estimate" given in Proposition 4.2 below.

Lemma 4.1. For all $\alpha \in(0,1)$ and $p \in(1, \infty)$

$$
\||f|\|_{\alpha, p} \lesssim\|f\|_{\alpha, p}
$$

Proof. It suffices to notice that, for every $x \in G, S_{\alpha}^{\text {loc }}(|f|)(x) \leq S_{\alpha}^{\text {loc }}(f)(x)$, and use the representation of the $L_{\alpha}^{p}$-norm given by Theorem 1.3 .

Proposition 4.2. Let $\alpha, \beta, \gamma \geq 0,1<p, r<\infty, 1<q \leq \infty$ and $0<\theta<1$ be such that $\gamma=\theta \alpha+(1-\theta) \beta$ and $1 / r=\theta / p+(1-\theta) / q$. Then for all $f \in L_{\alpha}^{p} \cap L_{\beta}^{q}$

$$
\|f\|_{\gamma, r} \lesssim\|f\|_{\alpha, p}^{\theta}\|f\|_{\beta, q}^{1-\theta} .
$$

Proof. Notice that it is enough to give the proof in the case when $\beta=0$.

We then take $\alpha, \gamma>0,1<p, r<\infty, 1<q \leq \infty$ and $0<\theta<1$ such that $\gamma=\theta \alpha$ and $1 / r=\theta / p+(1-\theta) / q$. By (1.1) we have $\|f\|_{\gamma, r}=\|f\|_{r}+\left\|\Delta^{\gamma / 2} f\right\|_{r}$. Choose $a, b, s$ such that 
$a+b=r, a s=p$ and $b s^{\prime}=q$. By Hölder's inequality we obtain that

$$
\|f\|_{r} \leq\left(\int_{G}|f|^{a s} \mathrm{~d} \rho\right)^{1 / s r}\left(\int_{G}|f|^{b s^{\prime}} \mathrm{d} \rho\right)^{1 / s^{\prime} r}=\|f\|_{p}^{a / r}\|f\|_{q}^{b / r}=\|f\|_{p}^{\theta}\|f\|_{q}^{1-\theta} \leq\|f\|_{\alpha, p}^{\theta}\|f\|_{q}^{1-\theta} .
$$

It remains to estimate $\left\|\Delta^{\gamma / 2} f\right\|_{r}$.

If $q<\infty$, choose $\delta>0$ and $k \in \mathbb{N}$ such that $\delta+\gamma / 2=k$. Then if $a=2 \theta\left(k-\frac{1}{2}-\frac{\alpha}{2}\right)$ and $b=2(1-\theta)\left(k-\frac{1}{2}\right)$, by applying Hölder's inequality in $t$ we get

$$
\begin{aligned}
\left(g_{\delta} \Delta^{\gamma / 2} f(x)\right)^{2} & =\int_{0}^{+\infty} t^{a+b}\left|\Delta^{k} T_{t} f(x)\right|^{2} \mathrm{~d} t \\
& \leq\left(g_{k-\alpha / 2} \Delta^{\alpha / 2} f(x)\right)^{2 \theta}\left(g_{k} f(x)\right)^{2(1-\theta)} .
\end{aligned}
$$

Therefore, by Littlewood-Paley-Stein theory, (4.12) and applying Hölder's inequality in the $x$-variable

$$
\begin{aligned}
\left\|\Delta^{\gamma / 2} f\right\|_{r} & \approx\left\|g_{\delta} \Delta^{\gamma / 2} f\right\|_{r} \\
& \leq\left(\int_{G}\left(g_{k-\alpha / 2} \Delta^{\alpha / 2} f(x)\right)^{\theta r}\left(g_{k} f(x)\right)^{(1-\theta) r} \mathrm{~d} \rho(x)\right)^{1 / r} \\
& \leq\left(\int_{G}\left(g_{k-\alpha / 2} \Delta^{\alpha / 2} f(x)\right)^{\theta r s} \mathrm{~d} \rho(x)\right)^{1 / s r}\left(\int_{G}\left(g_{k} f(x)\right)^{(1-\theta) r s^{\prime}} \mathrm{d} \rho(x)\right)^{1 / s^{\prime} r} \\
& =\left\|g_{k-\alpha / 2} \Delta^{\alpha / 2} f\right\|_{p}^{\theta}\left\|g_{k} f\right\|_{q}^{1-\theta} \\
& \lesssim\left\|\Delta^{\alpha / 2} f\right\|_{p}^{\theta}\|f\|_{q}^{1-\theta} \\
& \lesssim\|f\|_{\alpha, p}^{\theta}\|f\|_{q}^{1-\theta} .
\end{aligned}
$$

Estimates (4.11) and (4.13) prove the proposition in the case when $q$ is finite and $\beta=0$.

Suppose now that $q=\infty$. We follow closely [1, Theorem 2.4] using a complex interpolation argument.

Assume first that $f$ and $h$ are nonnegative simple functions and define for $z \in \Sigma_{0}^{\infty}=\{z \in$ $\mathbb{C}: \Re z \geq 0\}$

$$
w(z)=\int_{G} \Delta^{-\alpha z / 2} f(x) h(x)^{(1-1 / p)(1-z)+z} \mathrm{~d} \rho(x) .
$$

The function $w$ is continuous in $\Sigma_{0}^{\infty}$, holomorphic in the interior of $\Sigma_{0}^{\infty}$ and bounded in any strip $\Sigma_{0}^{c}=\{z \in \mathbb{C}: 0 \leq \Re z \leq c\}, c \in \mathbb{R}^{+}$. When $z=i \zeta, \zeta \in \mathbb{R}$, by [34 there exists a positive constant $C_{p}$ such that

$$
\left\|\Delta^{-i \alpha \zeta / 2} f\right\|_{p} \leq C_{p}(1+|\alpha \zeta| / 2)^{1 / 2} e^{\frac{\pi}{2} \frac{|\alpha \zeta|}{2}}\|f\|_{p},
$$

so that

$$
|w(i \zeta)| \leq C_{p}(1+|\alpha \zeta| / 2)^{1 / 2} e^{\frac{\pi}{2} \frac{|\alpha \zeta|}{2}}\|f\|_{p}\|h\|_{1}^{1-1 / p} .
$$


On the other hand, since $f$ is nonnegative

$$
\begin{aligned}
\left|\Delta^{-\alpha / 2-i \alpha \zeta / 2} f(x)\right| & =\frac{1}{|\Gamma(\alpha / 2+i \alpha \zeta / 2)|}\left|\int_{0}^{\infty} t^{\alpha / 2+i \alpha \zeta / 2-1} e^{-t \Delta} f(x) \mathrm{d} t\right| \\
& \leq \frac{1}{|\Gamma(\alpha / 2+i \alpha \zeta / 2)|} \int_{0}^{\infty} t^{\alpha / 2-1} e^{-t \Delta} f(x) \mathrm{d} t \\
& =\frac{\Gamma(\alpha / 2)}{|\Gamma(\alpha / 2+i \alpha \zeta / 2)|} \Delta^{-\alpha / 2} f(x) .
\end{aligned}
$$

Thus

$$
|w(1+i \zeta)| \leq \frac{\Gamma(\alpha / 2)}{|\Gamma(\alpha / 2+i \alpha \zeta / 2)|}\left\|\Delta^{-\alpha / 2} f\right\|_{\infty}\|h\|_{1} .
$$

Define $W(z)=\Gamma(1+\alpha z / 2) \frac{1}{1+z} w(z)$ for $z \in \Sigma_{0}^{\infty}$. By the estimates satisfied by the function $w$ on the boundary of the strip $\Sigma_{0}^{1}$ and the three lines theorem we get

$$
|W(1-\theta)| \lesssim\|f\|_{p}^{\theta}\left\|\Delta^{-\alpha / 2} f\right\|_{\infty}^{1-\theta}\|h\|_{1}^{(1-1 / p) \theta+1-\theta},
$$

which implies that

$$
|w(1-\theta)| \lesssim\|f\|_{p}^{\theta}\left\|\Delta^{-\alpha / 2} f\right\|_{\infty}^{1-\theta}\|h\|_{1}^{1 / r^{\prime}}
$$

By taking the supremum over all functions $g=h^{1 / r^{\prime}}$ such that $\|g\|_{r^{\prime}} \leq 1$ we obtain that

$$
\left\|\Delta^{-(1-\theta) \alpha / 2} f\right\|_{r} \lesssim\|f\|_{p}^{\theta}\left\|\Delta^{-\alpha / 2} f\right\|_{\infty}^{1-\theta}
$$

for all nonnegative functions $f$. This implies that

$$
\left\|\Delta^{\gamma / 2} g\right\|_{r} \lesssim\left\|\Delta^{\alpha / 2} g\right\|_{p}^{\theta}\|g\|_{\infty}^{1-\theta}
$$

for all nonnegative functions $g$. By (4.11) and the estimate above we deduce that for all nonnegative functions

$$
\|g\|_{\gamma, r} \lesssim\|g\|_{\alpha, p}^{\theta}\|g\|_{\infty}^{1-\theta}
$$

Take now $\alpha \in[0,1]$ and $f$ of arbitrary sign. Then writing $f=f_{+}-f_{-}$, applying (4.14) to $f_{+}$and $f_{-}$, using Lemma 4.1 and noticing that $\left\|\Delta^{\alpha / 2} f_{ \pm}\right\|_{p} \lesssim\left\|\Delta^{\alpha / 2} f\right\|_{p}$ we obtain that

$$
\|f\|_{\gamma, r} \lesssim\|f\|_{\alpha, p}^{\theta}\|f\|_{\infty}^{1-\theta}
$$

It remains to consider the case when $\alpha>1$. Suppose first that $\gamma<1, \theta \in(0,1), \alpha>1$, $\gamma=\theta \alpha$ and $\frac{1}{r}=\frac{\theta}{p}$. We choose $\beta<1$ such that $\gamma<\beta<\gamma r$ and $s>1$ such that $\frac{1}{r}=\frac{\gamma}{\beta s}$. Then by 4.15)

$$
\|f\|_{\gamma, r} \lesssim\|f\|_{\beta, s}^{\tilde{\theta}}\|f\|_{\infty}^{1-\tilde{\theta}}
$$

for $\tilde{\theta} \in(0,1)$ such that $\gamma=\tilde{\theta} \beta$ and $\frac{1}{r}=\frac{\tilde{\theta}}{s}$. Moreover, by (4.10)

$$
\|f\|_{\beta, s} \lesssim\|f\|_{\alpha, p}^{\theta^{\prime}}\|f\|_{\gamma, r}^{1-\theta^{\prime}}
$$

for $\theta^{\prime} \in(0,1)$ such that $\beta=\theta^{\prime} \alpha+\left(1-\theta^{\prime}\right) \gamma$, and $\frac{1}{s}=\frac{\theta^{\prime}}{p}+\frac{1-\theta^{\prime}}{r}$. Putting together the two estimates above we obtain

$$
\|f\|_{\gamma, r} \lesssim\|f\|_{\alpha, p}^{\theta}\|f\|_{\infty}^{1-\theta}
$$


which proves the theorem for $\alpha>1$ and $\gamma<1$. Take now $\gamma \geq 1$ and choose $q$ such that $\gamma r<q<r$ and $\beta<1$ such that $\beta q=\gamma r$. We have

$$
\|f\|_{\gamma, r} \lesssim\|f\|_{\alpha, p}^{\tilde{\theta}}\|f\|_{\beta, q}^{1-\tilde{\theta}}
$$

for $\gamma=\tilde{\theta} \alpha+(1-\tilde{\theta}) \beta$ and $\frac{1}{r}=\frac{\tilde{\theta}}{p}+\frac{1-\tilde{\theta}}{q}$. By (4.16) we get

$$
\|f\|_{\beta, q} \lesssim\|f\|_{\alpha, p}^{\theta^{\prime}}\|f\|_{\infty}^{1-\theta^{\prime}}
$$

where $\beta=\theta^{\prime} \alpha$ and $\frac{1}{q}=\frac{\theta^{\prime}}{p}$. Putting together the two estimates above we obtain

$$
\|f\|_{\gamma, r} \lesssim\|f\|_{\alpha, p}^{\theta}\|f\|_{\infty}^{1-\theta}
$$

which proves the theorem for $\alpha>1, \gamma \geq 1, q=\infty$ and $\beta=0$. The proof of the proposition is now complete.

4.2. A representation formula for the Sobolev norm in terms of $D_{\alpha}^{\text {loc }}$. Recall that in (1.3) we have defined the quantity $D_{\alpha}^{\text {loc }} f$. We shall prove Theorem 1.3 (ii). To do so, we first need some tools and some technical results that we shall introduce below.

For every locally integrable function $f$ and every ball $B$ we denote by $f_{B}$ the average $\frac{1}{\rho(B)} \int_{B} f \mathrm{~d} \rho$. For every $q \in[1, \infty), r>0, x \in G$ we define

$$
\Omega_{f}^{(q)}(x, r)=\sup \left\{\left(\frac{1}{\rho(B)} \int_{B}\left|f-f_{B}\right|^{q} \mathrm{~d} \rho\right)^{1 / q}: B \in \mathcal{B}_{r}, x \in B\right\}
$$

and

$$
\Omega_{f}^{\infty}(x, r)=\sup \left\{\left\|f-f_{B}\right\|_{\infty}: B \in \mathcal{B}_{r}, x \in B\right\} .
$$

We recall that $\mathcal{B}_{R}$ denotes the collection of balls of radius $\leq R$. We simply write $\Omega_{f}(x, r)$ for $\Omega_{f}^{(1)}(x, r)$.

Lemma 4.3. For every locally integrable function $f$ the following hold:

(i) $\Omega_{f}(x, r) \leq \Omega_{f}^{(q)}(x, r)$ for every $q \in[1, \infty], r>0$;

(ii) if $B, B^{\prime} \in \mathcal{B}_{1}$ and $B \subset B^{\prime}$, then

$$
\frac{1}{\rho(B)} \int_{B}\left|f-f_{B}\right| \mathrm{d} \rho \leq 2 \frac{\rho\left(B^{\prime}\right)}{\rho(B)} \frac{1}{\rho\left(B^{\prime}\right)} \int_{B^{\prime}}\left|f-f_{B^{\prime}}\right|^{q} \mathrm{~d} \rho
$$

(iii) for every $x \in G, r \leq s \leq 2 r \leq 2$

$$
\Omega_{f}(x, r) \lesssim \Omega_{f}(x, s) \lesssim \Omega_{f}(x, 2 r) ;
$$

(iv) for every $B \in \mathcal{B}$ of radius $r$ and almost every $y \in B$

$$
\left|f(y)-f_{B}\right| \lesssim \int_{0}^{8 r} \Omega_{f}(y, s) \frac{\mathrm{d} s}{s} .
$$

The above lemma was proved in [15]: the same proof works in our setting, since only the local doubling property plays a role here. 
For every locally integrable function $f, q \in[1, \infty], R>0, \alpha \in(0,1)$ and $x \in G$ we define

$$
\begin{aligned}
G_{\alpha}^{\mathrm{loc}} f(x) & =\left(\int_{0}^{1}\left[r^{-\alpha} \Omega_{f}(x, r)\right]^{2} \frac{\mathrm{d} r}{r}\right)^{1 / 2} \\
G_{\alpha, q}^{\mathrm{loc}} f(x) & =\left(\int_{0}^{1}\left[r^{-\alpha} \Omega_{f}^{(q)}(x, r)\right]^{2} \frac{\mathrm{d} r}{r}\right)^{1 / 2} \\
G_{\alpha}^{R} f(x) & =\left(\int_{0}^{R}\left[r^{-\alpha} \Omega_{f}(x, r)\right]^{2} \frac{\mathrm{d} r}{r}\right)^{1 / 2} \\
G_{\alpha, q}^{R} f(x) & =\left(\int_{0}^{R}\left[r^{-\alpha} \Omega_{f}^{(q)}(x, r)\right]^{2} \frac{\mathrm{d} r}{r}\right)^{1 / 2} \\
S_{\alpha}^{R} f(x) & =\left(\int_{0}^{R}\left[\frac{1}{u^{\alpha} V(u)} \int_{|y|<u}\left|f\left(x y^{-1}\right)-f(x)\right| \mathrm{d} \rho(y)\right]^{2} \frac{\mathrm{d} u}{u}\right)^{1 / 2} .
\end{aligned}
$$

Lemma 4.4. For every $R_{1}, R_{2}>0, p \in(1, \infty)$ and $\alpha \in(0,1)$,

$$
\begin{aligned}
\left\|S_{\alpha}^{R_{1}} f\right\|_{p}+\|f\|_{p} & \approx\left\|S_{\alpha}^{R_{2}} f\right\|_{p}+\|f\|_{p}, \\
\left\|G_{\alpha}^{R_{1}} f\right\|_{p}+\|f\|_{p} & \approx\left\|G_{\alpha}^{R_{2}} f\right\|_{p}+\|f\|_{p} .
\end{aligned}
$$

Proof. Assume $R_{1} \leq R_{2}$. Then it is obvious that $S_{\alpha}^{R_{1}} f(x) \leq S_{\alpha}^{R_{2}} f(x)$ and $G_{\alpha}^{R_{1}} f(x) \leq$ $G_{\alpha}^{R_{2}} f(x)$ for every $x \in G$, so that

$$
\begin{gathered}
\left\|S_{\alpha}^{R_{1}} f\right\|_{p}+\|f\|_{p} \leq\left\|S_{\alpha}^{R_{2}} f\right\|_{p}+\|f\|_{p} \\
\left\|G_{\alpha}^{R_{1}} f\right\|_{p}+\|f\|_{p} \leq\left\|G_{\alpha}^{R_{2}} f\right\|_{p}+\|f\|_{p} .
\end{gathered}
$$

In order to prove the lemma, using (2.2), we notice that

$$
\begin{aligned}
& \int_{R_{1}}^{R_{2}}\left[\frac{1}{r^{\alpha} V(r)} \int_{|y| \leq r}\left|f\left(x y^{-1}\right)-f(x)\right| \mathrm{d} \rho(y)\right]^{2} \frac{\mathrm{d} r}{r} \\
& =\int_{R_{1}}^{R_{2}}\left[\frac{1}{r^{\alpha} V(r)} \int_{|y| \leq r}|f(x y)-f(x)| \mathrm{d} \lambda(y)\right]^{2} \frac{\mathrm{d} r}{r} \\
& \lesssim \int_{R_{1}}^{R_{2}}\left[\frac{1}{r^{\alpha} V(r)} \int_{B(x, r)}|f(z)| \mathrm{d} \lambda(z)+\frac{|f(x)|}{r^{\alpha}}\right]^{2} \frac{\mathrm{d} r}{r} \\
& =\int_{R_{1}}^{R_{2}}\left[\frac{1}{r^{\alpha} V(r)} \int_{B(x, r)}|f(z)| \delta(z) \mathrm{d} \rho(z)+\frac{|f(x)|}{r^{\alpha}}\right]^{2} \frac{\mathrm{d} r}{r} \\
& \lesssim \int_{R_{1}}^{R_{2}}\left[\frac{1}{r^{\alpha} V(r)} \int_{B(x, r)}|f(z)| \delta(x) \mathrm{d} \rho(z)+\frac{|f(x)|^{2}}{r^{\alpha}}\right]^{2} \frac{\mathrm{d} r}{r} \\
& \lesssim \int_{R_{1}}^{R_{2}}\left[\frac{1}{r^{\alpha}} M^{R_{2}} f(x)+\frac{|f(x)|}{r^{\alpha}}\right]^{2} \frac{\mathrm{d} r}{r} \\
& \lesssim M^{R_{2}} f(x)+|f(x)|,
\end{aligned}
$$

where $M^{R_{2}}$ is the local maximal function defined in (2.10). It follows that

$$
\left\|S_{\alpha}^{R_{2}} f\right\|_{p}+\|f\|_{p} \leq\left\|S_{\alpha}^{R_{1}} f\right\|_{p}+\|f\|_{p}+\left\|M^{R_{2}} f\right\|_{p}+\|f\|_{p} \lesssim\left\|S_{\alpha}^{R_{1}} f\right\|_{p}+\|f\|_{p}
$$


where we have applied the boundedness of the maximal function $M^{R_{2}}$ on $L^{p}$. A similar argument shows that for every $r \leq R_{2}, \Omega_{f}(x, r) \leq M^{R_{2}} f(x)$ so that

$$
\int_{R_{1}}^{R_{2}}\left[r^{-\alpha} \Omega_{f}(x, r)\right]^{2} \frac{\mathrm{d} r}{r} \lesssim \int_{R_{1}}^{R_{2}}\left[M^{R_{2}} f(x)\right]^{2} \frac{\mathrm{d} r}{r^{1+\alpha}} .
$$

Thus

$$
\left\|G_{\alpha}^{R_{2}} f\right\|_{p}+\|f\|_{p} \lesssim\left\|G_{\alpha}^{R_{1}} f\right\|_{p}+\|f\|_{p}+\left\|M^{R_{2}} f\right\|_{p} \lesssim\left\|G_{\alpha}^{R_{1}} f\right\|_{p}+\|f\|_{p},
$$

applying again the $L^{p}$ boundedness of the maximal function $M^{R_{2}}$.

Proposition 4.5. For every locally integrable function $f, \alpha \in(0,1) p \in(1, \infty)$ and $R_{1}, R_{2}>0$ the following hold:

(i) if $p \in(1,2], \alpha \leq \frac{d}{p}, q<\frac{d p}{d-\alpha p}$, then

$$
\left\|G_{\alpha, q}^{R_{1}} f\right\|_{p}+\|f\|_{p} \approx\left\|G_{\alpha}^{R_{2}} f\right\|_{p}+\|f\|_{p} ;
$$

(ii) if $p \in[2, \infty), \alpha \leq \frac{d}{p}, q<\frac{2 d}{d-2 \alpha}$, then

$$
\left\|G_{\alpha, q}^{R_{1}} f\right\|_{p}+\|f\|_{p} \approx\left\|G_{\alpha}^{R_{2}} f\right\|_{p}+\|f\|_{p} ;
$$

(iii) if $p \in(1, \infty), \alpha>\frac{d}{p}$, then

$$
\left\|G_{\alpha, \infty}^{R_{1}} f\right\|_{p}+\|f\|_{p} \approx\left\|G_{\alpha}^{R_{2}} f\right\|_{p}+\|f\|_{p} .
$$

Proof. In view of Lemma 4.4 we can assume $R_{1}=R_{2}=R$. We shall prove (i). The proofs of statements (ii-iii) are similar and we omit them.

By Lemma 4.3(i) it follows that for every $p \in(1, \infty)$ and $q>1,\left\|G_{\alpha}^{R} f\right\|_{p} \lesssim\left\|G_{\alpha, q}^{R} f\right\|_{p}$.

For every ball $B^{\prime}=B\left(y^{\prime}, s\right)$ with $s<1$, and every $y \in B^{\prime}$ we have that

$$
\int_{B^{\prime}}\left|f-f_{B^{\prime}}\right| \mathrm{d} \rho \leq \int_{B^{\prime}} \Omega_{f}(z, s) \mathrm{d} \rho(z) \leq \int_{B(y, 2 s)} \Omega_{f}(z, s) \mathrm{d} \rho(z),
$$

so that by the local doubling property

$$
\frac{1}{\rho\left(B^{\prime}\right)} \int_{B^{\prime}}\left|f-f_{B^{\prime}}\right| \mathrm{d} \rho \lesssim \frac{1}{\rho(B(y, 2 s))} \int_{B(y, 2 s)} \Omega_{f}(z, s) \mathrm{d} \rho(z) .
$$

It follows that

$$
\Omega_{f}(y, s) \lesssim \frac{1}{\rho(B(y, 2 s))} \int_{B(y, 2 s)} \Omega_{f}(z, s) \mathrm{d} \rho(z) .
$$

By Lemma 4.3(iv) for every ball $B=B\left(y_{0}, r\right), r<1$, for almost every $y \in B$ and for every $\beta \geq 0$

$$
\begin{aligned}
\left|f(y)-f_{B}\right| & \lesssim \int_{0}^{8 r} \frac{1}{\rho(B(y, 2 s))} \int_{B(y, 2 s)} \Omega_{f}(z, s) \mathrm{d} \rho(z) \frac{\mathrm{d} s}{s} \\
& =\int_{0}^{8 r} \frac{\rho(B(y, 2 s))^{-\beta}}{\rho(B(y, 2 s))^{1-\beta}} \int_{B(y, 2 s)} \Omega_{f}(z, s) \chi_{3 B}(z) \mathrm{d} \rho(z) \frac{\mathrm{d} s}{s} \\
& \lesssim \int_{0}^{8 r}\left[\delta^{-1}(y) V(2 s)\right]^{-\beta} M_{\beta}^{1}\left(\Omega_{f}(\cdot, s) \chi_{3 B}\right)(y) \frac{\mathrm{d} s}{s} \\
& \lesssim \delta\left(y_{0}\right)^{\beta} \int_{0}^{8 r} V(s)^{-\beta} M_{\beta}^{1}\left(\Omega_{f}(\cdot, s) \chi_{3 B}\right)(y) \frac{\mathrm{d} s}{s}
\end{aligned}
$$


where we applied again the local doubling property and where $M_{\beta}^{1}$ is the modified local

maximal function defined in (2.11). Take now $p \in(1,2], \alpha \leq \frac{d}{p}, q<\frac{d p}{d-p \alpha}$. We choose $p_{0}<p$ and $0 \leq \beta<\frac{\alpha}{d} \leq \frac{1}{p}<1$ such that $\frac{1}{q}=\frac{1}{p_{0}}-\beta$. Then $M_{\beta}^{1}$ is bounded from $L^{p_{0}}$ to $L^{q}$. This implies that

$$
\begin{aligned}
& \frac{1}{\rho(B)^{1 / q}}\left\|f-f_{B}\right\|_{L^{q}(B)} \\
& \lesssim \frac{1}{\rho(B)^{1 / q}} \delta\left(y_{0}\right)^{\beta} \int_{0}^{8 r} V(s)^{-\beta}\left\|\Omega_{f}(\cdot, s)\right\|_{L^{p_{0}(3 B)}} \frac{\mathrm{d} s}{s} \\
& \leq \frac{1}{\rho(B)^{1 / q}} \delta\left(y_{0}\right)^{\beta} \rho(3 B)^{\frac{1}{p_{0}}} \int_{0}^{8 r} V(s)^{-\beta}\left(\frac{1}{\rho(3 B)} \int_{3 B}\left|\Omega_{f}(z, s)\right|^{p_{0}} \mathrm{~d} \rho(z)\right)^{\frac{1}{p_{0}}} \frac{\mathrm{d} s}{s} \\
& =\delta\left(y_{0}\right)^{\frac{1}{q}+\beta-\frac{1}{p_{0}}} V(r)^{-\frac{1}{q}+\frac{1}{p_{0}}} \int_{0}^{8 r} V(s)^{-\beta} M^{3}\left(\left|\Omega_{f}(\cdot, s)\right|^{p_{0}}\right)^{\frac{1}{p_{0}}}(x) \frac{\mathrm{d} s}{s} .
\end{aligned}
$$

Since $\frac{1}{q}=\frac{1}{p_{0}}-\beta$, we get

$$
\Omega_{f}^{(q)}(x, r) \lesssim V(r)^{\beta} \int_{0}^{8 r} V(s)^{-\beta} M^{3}\left(\left|\Omega_{f}(\cdot, s)\right|^{p_{0}}\right)^{\frac{1}{p_{0}}}(x) \frac{\mathrm{d} s}{s},
$$

and

$$
G_{\alpha, q}^{R} f(x) \leq\left(\int_{0}^{R} r^{-2 \alpha} V(r)^{2 \beta}\left[\int_{0}^{8 r} V(s)^{-\beta} M^{3}\left(\left|\Omega_{f}(\cdot, s)\right|^{p_{0}}\right)^{\frac{1}{p_{0}}} \frac{\mathrm{d} s}{s}\right]^{2} \frac{\mathrm{d} r}{r}\right)^{1 / 2} .
$$

Now arguing as in [15, p.318] the statement (i) follows.

We are now ready to prove the representation formula of the Sobolev norm involving the functional $D_{\alpha}^{\text {loc }}$.

Proof of Theorem 1.3 (ii). STEP I. We shall prove that

$$
\left\|S_{\alpha}^{\mathrm{loc}} f\right\|_{p} \lesssim\left\|D_{\alpha}^{\mathrm{loc}} f\right\|_{p} .
$$

Indeed, by (2.3) for every $x \in G$

$$
\begin{aligned}
S_{\alpha}^{\operatorname{loc}} f(x)^{2} & =\int_{0}^{1} \frac{1}{u^{2 \alpha} V(u)}\left(\int_{B_{u}}\left|f\left(x y^{-1}\right)-f(x)\right| \mathrm{d} \rho(y)\right)^{2} \frac{\mathrm{d} u}{u} \\
& \lesssim \int_{0}^{1} \frac{1}{u^{2 \alpha} V(u)} \int_{B_{u}}\left|f\left(x y^{-1}\right)-f(x)\right|^{2} \mathrm{~d} \rho(y) \frac{\mathrm{d} u}{u} \\
& =\int_{|y|<1}\left(\int_{|y|}^{1} \frac{1}{u^{2 \alpha+d+1}} \mathrm{~d} u\right)\left|f\left(x y^{-1}\right)-f(x)\right|^{2} \mathrm{~d} \rho(y) \\
& \lesssim D_{\alpha}^{\operatorname{loc}} f(x)^{2}
\end{aligned}
$$

so that $\left\|S_{\alpha}^{\text {loc }} f\right\|_{p} \lesssim\left\|D_{\alpha}^{\text {loc }} f\right\|_{p}$ and $\|f\|_{\alpha, p} \lesssim\left\|D_{\alpha}^{\text {loc }} f\right\|_{p}+\|f\|_{p}$.

STEP II. We shall prove that, for $p>2 d /(d+2 \alpha)$

$$
\left\|D_{\alpha}^{\mathrm{loc}} f\right\|_{p} \lesssim\left\|S_{\alpha}^{\mathrm{loc}} f\right\|_{p}+\|f\|_{p} .
$$

By applying Proposition 4.5 and Theorem 1.3 (i) it is enough to prove that

$$
D_{\alpha}^{\mathrm{loc}} f(x) \lesssim G_{\alpha, 2}^{\mathrm{loc}} f(x),
$$


and

$$
\left\|G_{\alpha}^{\mathrm{loc}} f\right\|_{p} \lesssim\left\|S_{\alpha}^{\mathrm{loc}} f\right\|_{p}+\|f\|_{p} .
$$

Indeed, we observe that $G_{\alpha, 2}^{\text {loc }} f(x) \leq G_{\alpha, \infty}^{\text {loc }} f(x)$ and apply Proposition 4.5 (iii) if $\alpha>d / p$, and Proposition 4.5 (i-ii) with $q=2$ if $\alpha \leq d / p$. In this latter case, we need to assume $p>2 d /(d+2 \alpha)$. To prove (4.18) we argue as follows:

$$
\begin{aligned}
D_{\alpha}^{\mathrm{loc}} f(x)^{2} & =\sum_{k=-\infty}^{-1} \int_{2^{k-1} \leq|y|<2^{k}} \frac{\left|f\left(x y^{-1}\right)-f(x)\right|^{2}}{|y|^{2 \alpha} V(|y|)} \mathrm{d} \rho(y) \\
& \lesssim \sum_{k=-\infty}^{-1} \frac{1}{2^{2 k \alpha} V\left(2^{k}\right)} \int_{|y|<2^{k}}\left|f\left(x y^{-1}\right)-f(x)\right|^{2} \mathrm{~d} \rho(y) \\
& \lesssim \int_{0}^{1} \frac{1}{r^{2 \alpha} V(r)} \int_{|y|<r}\left|f\left(x y^{-1}\right)-f(x)\right|^{2} \mathrm{~d} \rho(y) \frac{\mathrm{d} r}{r} \\
& \lesssim \int_{0}^{1} \frac{1}{r^{2 \alpha} V(r)} \int_{B(x, r)}|f(z)-f(x)|^{2} \mathrm{~d} \lambda(z) \frac{\mathrm{d} r}{r} \\
& \lesssim \int_{0}^{1} \frac{\delta(x)}{r^{2 \alpha} V(r)} \int_{B(x, r)}\left|f(z)-f_{B(x, r)}\right|^{2} \mathrm{~d} \rho(z) \frac{\mathrm{d} r}{r} \\
& +\int_{0}^{1} \frac{1}{r^{2 \alpha} V(r)} \lambda(B(x, r))\left|f(x)-f_{B(x, r)}\right|^{2} \frac{\mathrm{d} r}{r} .
\end{aligned}
$$

We apply Lemma 4.3 (iv) and the following version of Hardy's inequality: If $g \geq 0$, $g \in L^{1}[0, R], R>0,1-p<\beta<1$ and $G(r)=\int_{0}^{r} g(t) \mathrm{d} t$, then,

$$
\int_{0}^{R} \frac{1}{r^{\beta}}\left(\frac{G(r)}{r}\right)^{p} \mathrm{~d} r \lesssim \int_{0}^{R} \frac{1}{r^{\beta}} g(r)^{p} \mathrm{~d} r
$$

We obtain

$$
\begin{aligned}
D_{\alpha}^{\text {loc }} f(x)^{2} & \lesssim \int_{0}^{1} \frac{1}{r^{2 \alpha+1}}\left[\Omega_{f}^{2}(x, r)\right]^{2} \mathrm{~d} r+\int_{0}^{1} \frac{1}{r^{2 \alpha+1}}\left[\int_{0}^{8 r} \Omega_{f}^{2}(x, u) \frac{\mathrm{d} u}{u}\right]^{2} \mathrm{~d} r \\
& \leq G_{\alpha, 2}^{1} f(x)^{2}+\int_{0}^{8} \frac{1}{u^{2 \alpha+1}}\left[\Omega_{f}^{2}(x, u)\right]^{2} \mathrm{~d} u \\
& \lesssim G_{\alpha, 2}^{8} f(x)^{2} .
\end{aligned}
$$

We shall now prove (4.19). For every $B\left(c_{B}, r\right)$, with $r \in(0,1], c_{B} \in G$ and $x \in B$

$$
\begin{aligned}
\int_{B}\left|f(y)-f_{B}\right| \mathrm{d} \rho(y) & \leq \int_{B}|f(y)-f(x)| \mathrm{d} \rho(y)+\int_{B}\left|f(x)-f_{B}\right| \mathrm{d} \rho(y) \\
& \leq 2 \int_{B}|f(y)-f(x)| \mathrm{d} \rho(y) .
\end{aligned}
$$

Using the fact that $\rho\left(B\left(c_{B}, r\right)\right)=\delta\left(c_{B}\right)^{-1} V(r) \approx \delta(x)^{-1} V(r)$, we deduce that

$$
\Omega_{f}(x, r) \lesssim \frac{2}{\delta(x)^{-1} V(r)} \int_{B(x, 2 r)}|f-f(x)| \mathrm{d} \rho,
$$


so that

$$
\begin{aligned}
\int_{0}^{1} r^{-2 \alpha-1}\left[\Omega_{f}(x, r)\right]^{2} \mathrm{~d} r & \lesssim \int_{0}^{1} r^{-2 \alpha}\left[\delta(x) V(r)^{-1} \int_{B(x, 2 r)}|f(y)-f(x)| \mathrm{d} \rho(y)\right]^{2} \frac{\mathrm{d} r}{r} \\
& \lesssim \int_{0}^{1} r^{-2 \alpha}\left[\delta(x) V(r)^{-1} \int_{|w|<2 r}|f(x w)-f(x)| \delta^{-1}(x w) \mathrm{d} \lambda(w)\right]^{2} \frac{\mathrm{d} r}{r} \\
& =\int_{0}^{1} r^{-2 \alpha}\left[V(r)^{-1} \int_{|w|<2 r}|f(x w)-f(x)| \mathrm{d} \rho(w)\right]^{2} \frac{\mathrm{d} r}{r} \\
& =\int_{0}^{1} r^{-2 \alpha}\left[V(r)^{-1} \int_{|w|<2 r}\left|f\left(x w^{-1}\right)-f(x)\right| \delta(w) \mathrm{d} \rho(w)\right]^{2} \frac{\mathrm{d} r}{r} \\
& \lesssim\left[S_{\alpha}^{2} f(x)\right]^{2},
\end{aligned}
$$

where we used the fact that the modular function is bounded on $B_{2 r}$. It follows that

$$
\left\|G_{\alpha}^{\mathrm{loc}} f\right\|_{p} \lesssim\left\|S_{\alpha}^{2} f\right\|_{p} \lesssim\left\|S_{\alpha}^{\mathrm{loc}} f\right\|_{p}+\|f\|_{p},
$$

as required in (4.19). This concludes the proof.

\section{Proof of Theorem 1.2}

We first prove Theorem 1.2 for $\alpha \in[0,1)$. The case when $\alpha=0$ is trivial. Suppose that

$\alpha \in(0,1), p_{1}, q_{2} \in(1, \infty]$ and $r, p_{2}, q_{1} \in(1, \infty)$ are such that $\frac{1}{r}=\frac{1}{p_{i}}+\frac{1}{q_{i}}, i=1,2$. Take $f \in L^{p_{1}} \cap L_{\alpha}^{p_{2}}$ and $g \in L^{q_{2}} \cap L_{\alpha}^{q_{1}}$. According to Theorem 1.3

$$
\|f g\|_{\alpha, r} \lesssim\left\|S_{\alpha}^{\mathrm{loc}}(f g)\right\|_{r}+\|f g\|_{r} .
$$

By Hölder's inequality one has

$$
\|f g\|_{r} \leq\|f\|_{p_{1}}\|g\|_{q_{1}} \leq\|f\|_{p_{1}}\|g\|_{\alpha, q_{1}},
$$

and

$$
\|f g\|_{r} \leq\|f\|_{p_{2}}\|g\|_{q_{2}} \leq\|f\|_{\alpha, p_{2}}\|g\|_{q_{2}}
$$

Moreover,

$$
\begin{aligned}
S_{\alpha}^{\operatorname{loc}}(f g)(x) \leq & \left(\int_{0}^{1}\left[\frac{1}{u^{\alpha} V(u)} \int_{|y|<u}\left|(f g)\left(x y^{-1}\right)-g\left(x y^{-1}\right) f(x)\right| \mathrm{d} \rho(y)\right]^{2} \frac{\mathrm{d} u}{u}\right)^{1 / 2} \\
& +\left(\int_{0}^{1}\left[\frac{1}{u^{\alpha} V(u)} \int_{|y|<u}\left|f(x) g\left(x y^{-1}\right)-(f g)(x)\right| \mathrm{d} \rho(y)\right]^{2} \frac{\mathrm{d} u}{u}\right)^{1 / 2} \\
= & I(x)+I I(x) .
\end{aligned}
$$

Obviously,

$$
I I(x)=\left|f(x) S_{\alpha}^{\text {loc }} g(x)\right|,
$$

so that by Hölder's inequality

$$
\|I I\|_{r} \leq\|f\|_{p_{1}}\left\|S_{\alpha}^{\mathrm{loc}} g\right\|_{q_{1}} \lesssim\|f\|_{p_{1}}\|g\|_{\alpha, q_{1}} .
$$


To estimate $I(x)$ we choose $p, q>1$ such that $q=p^{\prime}, 1<p<q_{2}$ and $p_{2}>\frac{q d}{d+q \alpha}$. By Hölder's inequality we obtain

$$
\begin{aligned}
& I(x) \lesssim\left(\int_{0}^{1}\left[\frac{1}{V(u)} \int_{|y|<u}\left|g\left(x y^{-1}\right)\right|^{p} \mathrm{~d} \rho(y)\right]^{2 / p}\right. \\
& \left.\times\left[\frac{1}{V(u)} \int_{|y|<u}\left|f\left(x y^{-1}\right)-f(x)\right|^{q} \mathrm{~d} \rho(y)\right]^{2 / q} \frac{\mathrm{d} u}{u^{2 \alpha+1}}\right)^{1 / 2} \\
& =\left(\int_{0}^{1}\left[\frac{1}{V(u)} \int_{|y|<u}|g(x y)|^{p} \mathrm{~d} \lambda(y)\right]^{2 / p}\left[\frac{1}{V(u)} \int_{|y|<u}|f(x y)-f(x)|^{q} \mathrm{~d} \lambda(y)\right]^{2 / q} \frac{\mathrm{d} u}{u^{2 \alpha+1}}\right)^{1 / 2} \\
& =\left(\int_{0}^{1}\left[\frac{1}{V(u)} \int_{B(x, u)}|g(z)|^{p} \mathrm{~d} \lambda(z)\right]^{2 / p}\left[\frac{1}{V(u)} \int_{B(x, u)}|f(z)-f(x)|^{q} \mathrm{~d} \lambda(z)\right]^{2 / q} \frac{\mathrm{d} u}{u^{2 \alpha+1}}\right)^{1 / 2} \\
& \lesssim\left(\int_{0}^{1}\left[\frac{\delta(x)}{V(u)} \int_{B(x, u)}|g(z)|^{p} \mathrm{~d} \rho(z)\right]^{2 / p}\left[\frac{\delta(x)}{V(u)} \int_{B(x, u)}|f(z)-f(x)|^{q} \mathrm{~d} \rho(z)\right]^{2 / q} \frac{\mathrm{d} u}{u^{2 \alpha+1}}\right)^{1 / 2} \\
& \lesssim\left(M^{1}\left(|g|^{p}\right)(x)\right)^{1 / p}\left(\int_{0}^{1}\left[\frac{\delta(x)}{V(u)} \int_{B(x, u)}|f(z)-f(x)|^{q} \mathrm{~d} \rho(z)\right]^{2 / q} \frac{\mathrm{d} u}{u^{2 \alpha+1}}\right)^{1 / 2} \\
& \leq\left(M^{1}\left(|g|^{p}\right)(x)\right)^{1 / p} G_{\alpha, q}^{8} f(x) .
\end{aligned}
$$

The last inequality follows as in [15, p. 322-323].

Therefore, the boundedness properties of the local maximal function, Proposition 4.5, formula (4.19), Theorem 1.3 (i), and Hölder's inequality imply that

$$
\|I\|_{r} \leq\left\|\left(M^{1}\left(|g|^{p}\right)(x)\right)^{1 / p}\right\|_{q_{2}}\left(\left\|G_{\alpha, q}^{\mathrm{loc}} f\right\|_{p_{2}}+\|f\|_{p_{2}}\right) \lesssim\|g\|_{q_{2}}\|f\|_{\alpha, p_{2}} .
$$

In conclusion,

$$
\left\|S_{\alpha}^{\mathrm{loc}}(f g)\right\|_{r} \lesssim\|f\|_{p_{1}}\|g\|_{\alpha, q_{1}}+\|g\|_{q_{2}}\|f\|_{\alpha, p_{2}}
$$

as required to prove Theorem 1.2 for $\alpha \in(0,1]$.

We now prove Theorem 1.2 for $\alpha>1$. To do so, we argue by induction. Suppose that the theorem holds for a certain $\alpha>0$ : we shall show that it holds for $\beta=\alpha+1$. According to Proposition 3.2(ii)

$$
\|f g\|_{\beta, r} \approx\|f g\|_{\alpha, r}+\sum_{i=1}^{q}\left\|X_{i}(f g)\right\|_{\alpha, r} .
$$

On the one hand, by the inductive hypothesis

$$
\|f g\|_{\alpha, r} \lesssim\|f\|_{p_{1}}\|g\|_{\alpha, q_{1}}+\|f\|_{\alpha, p_{2}}\|g\|_{q_{2}} \leq\|f\|_{p_{1}}\|g\|_{\beta, q_{1}}+\|f\|_{\beta, p_{2}}\|g\|_{q_{2}} .
$$

On the other hand, for every $i=1, \ldots, q$,

$$
\left\|X_{i}(f g)\right\|_{\alpha, r} \leq\left\|\left(X_{i} f\right) g\right\|_{\alpha, r}+\left\|f\left(X_{i} g\right)\right\|_{\alpha, r} .
$$

Using the inductive hypothesis,

$$
\left\|f\left(X_{i} g\right)\right\|_{\alpha, r} \lesssim\|f\|_{\alpha, p_{3}}\left\|X_{i} g\right\|_{q_{3}}+\left\|X_{i} g\right\|_{\alpha, q_{1}}\|f\|_{p_{1}} \leq\|f\|_{\alpha, p_{3}}\left\|X_{i} g\right\|_{q_{3}}+\|g\|_{\beta, q_{1}}\|f\|_{p_{1}},
$$

where $\frac{1}{p_{3}}=\frac{\alpha}{\beta p_{2}}+\frac{1}{\beta p_{1}}$ and $\frac{1}{q_{3}}=\frac{\alpha}{\beta q_{2}}+\frac{1}{\beta q_{1}}$. One checks that $\frac{1}{p_{3}}+\frac{1}{q_{3}}=\frac{1}{r}$. By Proposition 4.2

$$
\|f\|_{\alpha, p_{3}} \lesssim\|f\|_{\beta, p_{2}}^{\alpha / \beta}\|f\|_{p_{1}}^{1-\alpha / \beta}
$$


and by Theorem 1.4 there exists $c$ sufficiently large such that

$$
\left\|X_{i} g\right\|_{q_{3}} \lesssim\left\|(c I+\Delta)^{1 / 2} g\right\|_{q_{3}} \sim\|g\|_{1, q_{3}} \lesssim\|g\|_{\beta, q_{1}}^{1 / \beta}\|g\|_{q_{2}}^{1-1 / \beta}
$$

where we applied Corollary 3.2(iii) and Proposition 4.2. It follows that

$$
\|f\|_{\alpha, p_{3}}\left\|X_{i} g\right\|_{q_{3}} \lesssim\left(\|f\|_{\beta, p_{2}}\|g\|_{q_{2}}\right)^{\alpha / \beta}\left(\|g\|_{\beta, q_{1}}\|f\|_{p_{1}}\right)^{1 / \beta} \lesssim\|f\|_{\beta, p_{2}}\|g\|_{q_{2}}+\|f\|_{p_{1}}\|g\|_{\beta, q_{1}} .
$$

In conclusion,

$$
\left\|f\left(X_{i} g\right)\right\|_{\alpha, r} \lesssim\|f\|_{\beta, p_{2}}\|g\|_{q_{2}}+\|f\|_{p_{1}}\|g\|_{\beta, q_{1}}
$$

as required. The term $\left\|g\left(X_{i} f\right)\right\|_{\alpha, r}$ can be treated in the similar way, so that the proof of the induction argument is complete and the theorem is proved for every $\alpha \geq 0$.

\section{FinAL REMARKS}

As we mentioned in the Introduction, we shall apply our main result Theorem 1.1 to the problem of well-posedness and regularity for solutions of the Cauchy problem for certain nonlinear differential equations involving the subLaplacian $\Delta$ on $G$, such as the heat and Schrödinger equations, see [11].

We would like to point out that our results, if on one hand solve the question of when $L_{\alpha}^{p} \cap L^{\infty}$ is an algebra on a generic Lie group, on the other hand leave open several interesting questions.

First of all, given the (counter)example in Theorem 3.3, it is certainly worth investigating the analogous of the results in the present paper in the case of the weighted Lebesgue and Sobolev spaces $L_{\alpha}^{p}\left(\delta^{\gamma}\right)$. This kind of weights arise naturally when considering the Sobolev embedding theorem (see [50]). Moreover, the spaces $L_{\alpha}^{p}\left(\delta^{\gamma}\right)$ might turn out to be the correct spaces for the well-posedness of some Cauchy problems - see [3], where Strichartz estimates involving such weighted Lebesgue spaces are proved for the Schrödinger equation associated with $\Delta$ on a class of Lie groups of exponential growth.

Finally, we mention that on a generic Lie group $G$, the $L^{p}$-boundedness of the Riesz transforms $\mathcal{R}_{j}, j=1, \ldots, q$, is not known, while it is known that higher order Riesz transforms might be unbounded (see the Introduction). These problems are connected with the study of the analogue of Theorems 1.1 and 1.2 for the homogeneous Sobolev spaces in our setting, which would be another interesting problem to investigate in the context of nonunimodular Lie groups.

\section{REFERENCES}

[1] D. R. Adams, N. G. Meyers, Bessel potentials. Inclusion relations among classes of exceptional sets, Indiana Univ. Math. J. 22 (1972/73), 873-905.

[2] G. Alexopoulos, An application of homogenization theory to harmonic analysis: Harnack inequalities and Riesz transforms on Lie groups of polynomial growth, Canad. J. Math. 44 (1992), 691-727.

[3] J.-Ph. Anker, V. Pierfelice, M. Vallarino, Schrödinger equations on Damek-Ricci spaces, Comm. Partial Differential Equations 36 (2011), no. 6, 976-997.

[4] P. Auscher, T. Coulhon, Riesz transform on manifolds and Poincaré inequalities, Ann. Sc. Norm. Super. Pisa Cl. Sci. (5) 4 (2005), no. 3, 531-555.

[5] P. Auscher, T. Coulhon, X. T. Duong, S. Hofmann, Riesz transform on manifolds and heat kernel regularity, Ann. Sci. École Norm. Sup. (4) 37 (2004), no. 6, 911-957. 
[6] N. Badr, F. Bernicot, E. Russ, Algebra properties for Sobolev spaces and applications to semilinear PDEs on manifolds, J. Anal. Math. 118 (2012), no. 2, 509-544.

[7] D. Bakry, Étude des transformations de Riesz dans les variétés riemanniennes á courbure de Ricci minorée, in: Séminaire de Probabilités XXI, in: Lecture Notes, vol. 1247, Springer, Berlin, 1987, pp. 137-172.

[8] J. Bergh, J. Löfström, Interpolation spaces. An introduction, Grundlehren der Mathematischen Wissenschaften, No. 223. Springer-Verlag, Berlin-New York, 1976.

[9] F. Bernicot, T. Coulhon, D. Frey, Sobolev algebras through heat kernel estimates, J. Éc. Polytech. Math. 3 (2016), 99-161.

[10] G. Bohnke, Algebres de Sobolev sur certains groupes nilpotents, J. Funct. Anal. 63 (1985), no. 3, $322-343$.

[11] T. Bruno, M. M. Peloso, A. Tabacco, M. Vallarino, Sobolev spaces on Lie groups: embedding theorems and algebra properties, submitted, arXiv:1804.10154,

[12] A. Carbonaro, O. Dragicević, Bellman function and linear dimension-free estimates in a theorem of Bakry, J. Funct. Anal. 265 (2013), no. 7, 1085-1104.

[13] A. Carbonaro, G. Mauceri, and S. Meda, $H^{1}$ and BMO for certain locally doubling metric measure spaces, Ann. Sc. Norm. Super. Pisa Cl. Sci. (5) 8 (2009), 543-582.

[14] Comparison of spaces of Hardy type for the Ornstein-Uhlenbeck operator, Potential Anal. 33 (2010), 85-105.

[15] T. Coulhon, E. Russ, V. Tardivel-Nachef, Sobolev algebras on Lie groups and Riemannian manifolds, Amer. J. Math. 123 (2001), no. 2, 283-342.

[16] M. Cowling, G. Gaudry, S. Giulini, G. Mauceri, Weak type $(1,1)$ estimates for heat kernel maximal functions on Lie groups, Trans. Amer. Math. Soc. 323 (1991), no. 2, 637-649.

[17] A. F. M. ter Elst, D. W. Robinson, Weighted subcoercive operators on Lie groups, J. Funct. Anal. 157 (1998), no. 1, 88-163.

[18] A. F. M. ter Elst, D. W. Robinson, A. Sikora, Heat kernels and Riesz transforms on nilpotent Lie groups, Colloq. Math. 74 (1997), no. 2, 191-218.

[19] G.B. Folland, Subelliptic estimates and function spaces on nilpotent Lie groups, Ark. Mat. 13 (1975), 161-207.

[20] I. Gallagher, Y. Sire, Besov algebras on Lie groups of polynomial growth, Studia Math. 212 (2012), no. 2, 119-139.

[21] G.I. Gaudry, T. Qian, P. Sjögren, Singular integrals associated to the Laplacian on the affine group $a x+b$, Ark. Mat. 30 (1992), no. 2, 259-281.

[22] D. Goldberg, A local version of real Hardy spaces, Duke Math. J. 46 (1979), no. 1, $27-42$.

[23] A. Grigor'yan, Upper bounds of derivatives of the heat kernel on an arbitrary complete manifold, J. Funct. Anal. 127 (1995), no. 2, 363-389.

[24] Y. Guivarc'h, Croissance polynomiale et priodes des fonctions harmoniques, Bull. Soc. Math. France 101 (1973), 333-379.

[25] W. Hebisch, G. Mauceri, S. Meda, Spectral multipliers for Sub-Laplacians with drift on Lie groups, Math. Z. 251 (2005), no. 4, 899-927.

[26] W. Hebisch, T. Steger, Multipliers and singular integrals on exponential growth groups, Math. Z. 245 (2003), no. 1, 37-61.

[27] T. Kato, G. Ponce, Commutator estimates and the Euler and Navier-Stokes equations, Comm. Pure Appl. Math. 41 (1988), no. 7, 891-907.

[28] H. Komatsu, Fractional powers of operators, Pacific J. Math. 19 (1966), 285-346.

[29] _ Fractional powers of operators. VI. Interpolation of non-negative operators and imbedding theorems, J. Fac. Sci. Univ. Tokyo Sect. IA Math. 19 (1972), 1-63.

[30] N. Lohoué, Transformées de Riesz et fonctions de Littlewood-Paley sur les groupes non moyennables, C. R. Acad. Sci. Paris Sr. I Math. 306 (1988), no. 7, 327-330.

[31] N. Lohoué, S. Mustapha, Sur les transformées de Riesz sur les groupes de Lie moyennables et sur certains espaces homogenes, Canad. J. Math. 50 (1998), no. 5, 1090-1104.

[32] N. Lohoué, N. Th. Varopoulos, Remarques sur les transformées de Riesz sur les groupes de Lie nilpotents, C. R. Acad. Sci. Paris Ser. I 301 (1985), 559-560. 
[33] G. Mauceri, S. Meda, M. Vallarino, Sharp endpoint results for imaginary powers and Riesz transforms on certain noncompact manifolds, Studia Math. 224 (2014), no. 2, 153-168.

[34] S. Meda, On the Littlewood-Paley-Stein g-function, Trans. Amer. Math. Soc. 347 (1995), no. 6, 2201-2212.

[35] S. Meda, S. Volpi, Spaces of Goldberg type on certain measured metric spaces, Ann. Mat. Pura Appl. (4) 196 (2017), no. 3, 947-981.

[36] D.W. Robinson, Elliptic operators and Lie groups, Oxford Mathematical Monographs. Oxford Science Publications. The Clarendon Press, Oxford University Press, New York, 1991.

[37] E. Russ, $H^{1}-L^{1}$ boundedness of Riesz transforms on Riemannian manifolds and on graphs, Potential Anal. 14 (2001), no. 3, 301-330.

[38] L. Saloff-Coste, Parabolic Harnack inequality for divergence form second order differential operators, Potential Anal. 4(4) (1995), 429-467.

[39] P. Sjögren, An estimate for a first-order Riesz operator on the affine group., Trans. Amer. Math. Soc. 351 (1999), no. 8, 3301-3314.

[40] P. Sjögren, M. Vallarino, Boundedness from $H^{1}$ to $L^{1}$ of Riesz transforms on a Lie group of exponential growth, Ann. Inst. Fourier (Grenoble) 58 (2008), no. 4, 1117-1151.

[41] E. M. Stein, Topics in harmonic analysis related to the Littlewood-Paley theory, Annals of Mathematics Studies, No. 63 Princeton University Press, Princeton, N.J.; University of Tokyo Press, Tokyo 1970.

[42] Singular integrals and differentiability properties of functions, Princeton Mathematical Series, No. 30 Princeton University Press, Princeton, N.J. 1970.

[43] R. Strichartz, Analysis of the Laplacian on the complete Riemannian manifold, J. Funct. Anal. 52 (1983) 48-79.

[44] _ Multipliers on fractional Sobolev spaces, J. Math. Mech. 16 (1967) 1031-1060.

[45] T. Tao, Nonlinear dispersive equations. Local and global analysis, CBMS Regional Conference Series in Mathematics, 106. Published for the Conference Board of the Mathematical Sciences, Washington, DC; by the American Mathematical Society, Providence, RI, 2006.

[46] M. Taylor, Hardy spaces and bmo on manifolds with bounded geometry, J. Geometric Anal. 19 (2009), 137-190.

[47] A. F. M. ter Elst, D. W. Robinson, Subcoercivity and subelliptic operators on Lie groups I. Free nilpotent groups. Potential Anal. 3 (1994), no. 3, 283-337.

[48] H. Triebel, Theory of function spaces. II, Monographs in Mathematics, 84. Birkhuser Verlag, Basel, 1992.

[49] N. Th. Varopoulos, Analysis on Lie groups, J. Funct. Anal. 76 (1988), no. 2, 346-410.

[50] Sobolev inequalities on Lie groups and symmetric spaces, J. Funct. Anal. 86 (1989), no. $1,19-40$.

[51] N. Th. Varopoulos, T. Coulhon, L. Saloffe-Coste, Analysis and geometry on groups, Cambridge Tracts in Mathematics, 100. Cambridge University Press, Cambridge, 1992.

Dipartimento di Matematica, Università degli Studi di Milano, Via C. Saldini 50, 20133 Milano, Italy

E-mail address: marco.peloso@unimi.it

Dipartimento di Scienze Matematiche "Giuseppe Luigi Lagrange", Dipartimento di Eccellenza 2018-2022, Politecnico di Torino, Corso Duca Degli Abruzzi 24, 10129 Torino, Italy

E-mail address: maria.vallarino@polito.it 